\title{
The Solar Isotope Spectrometer for the Advanced Composition Explorer
}

\author{
E. C. Stone, C. M. S. Cohen, W. R. Cook, A. C. Cummings, B. Gauld, \\ B. Kecman, R. A. Leske, R. A. Mewaldt and M. R. Thayer \\ California Institute of Technology, Pasadena, CA 91125
}

B. L. Dougherty, ${ }^{*}$ R. L. Grumm, B. D. Milliken, ${ }^{\dagger}$ R. G. Radocinski and

M. E. Wiedenbeck

Jet Propulsion Laboratory, Pasadena, CA 91109

E. R. Christian, S. Shuman and T. T. von Rosenvinge

NASA/Goddard Space Flight Center, Greenbelt, MD 20771

(Received ..... ; Accepted in final form .....)

\begin{abstract}
.
The Solar Isotope Spectrometer (SIS), one of nine instruments on the Advanced Composition Explorer (ACE), is designed to provide high resolution measurements of the isotopic composition of energetic nuclei from $\mathrm{He}$ to $\mathrm{Zn}(\mathrm{Z}=2$ to 30$)$ over the energy range from $\sim 10$ to $\sim 100 \mathrm{MeV} /$ nuc. During large solar events SIS will measure the isotopic abundances of solar energetic particles to determine directly the composition of the solar corona and to study particle acceleration processes. During solar quiet times SIS will measure the isotopes of low-energy cosmic rays from the Galaxy and isotopes of the anomalous cosmic ray component, which originates in the nearby interstellar medium. SIS has two telescopes composed of silicon solid-state detectors that provide measurements of the nuclear charge, mass, and kinetic energy of incident nuclei. Within each telescope, particle trajectories are measured with a pair of two-dimensional silicon strip detectors instrumented with custom very-largescale integrated (VLSI) electronics to provide both position and energy-loss measurements. SIS was especially designed to achieve excellent mass resolution under the extreme, high flux conditions encountered in large solar particle events. It provides a geometry factor of $\sim 40$ $\mathrm{cm}^{2} \mathrm{sr}$, significantly greater than earlier solar particle isotope spectrometers. A microprocessor controls the instrument operation, sorts events into prioritized buffers on the basis of their charge, range, angle of incidence, and quality of trajectory determination, and formats data for read out by the spacecraft. This paper describes the design and operation of SIS and the scientific objectives that the instrument will address.
\end{abstract}

Key words: Solar Energetic Particles, Anomalous Cosmic Rays, Galactic Cosmic Rays, Isotopes, Instrumentation

\section{Introduction}

The Advanced Composition Explorer (ACE) will perform comprehensive studies of the elemental, isotopic, and ionic charge state composition of energetic nuclei from $\mathrm{H}$ to $\mathrm{Zn}(1 \leq \mathrm{Z} \leq 30)$ from a vantage point located $\sim 1.5$ $\times 10^{6} \mathrm{~km}$ sunward of Earth, near the L1 libration point (Stone et al., 1998a).

\footnotetext{
* Present address: Mail Code 264-33, Caltech, Pasadena, CA 91125

$\dagger$ Present address: Lucent Technologies, Naperville, IL 60566
} 
These observations will address a wide range of questions concerning the origin, acceleration, and transport of energetic nuclei from solar, interplanetary, and galactic sources. ACE includes six high-resolution spectrometers and three interplanetary monitoring instruments that together will measure composition at energies ranging from $<1 \mathrm{keV} /$ nucleon (characteristic of the solar wind) to $\sim 500 \mathrm{MeV} /$ nucleon (characteristic of galactic cosmic radiation). The Solar Isotope Spectrometer (SIS), shown in Figure 1, is one of these six spectrometers and is designed to measure the elemental and isotopic composition over the energy range from $\sim 10$ to $\sim 100 \mathrm{MeV} /$ nuc, with excellent mass resolution and collecting power.

The primary objective of the ACE mission will be to determine and compare the elemental and isotopic composition of several distinct samples of matter, including the solar corona, the interplanetary medium, the local interstellar medium, and galactic matter. ACE will study matter from the Sun by measuring solar wind and solar energetic particles (SEPs). Matter from the local interstellar medium (LISM) will be observed at energies of $\sim 1$ $\mathrm{keV} /$ nucleon as interstellar neutral particles which enter the heliosphere and are ionized to become solar wind "pickup ions". Some of these ions are also observable as "anomalous cosmic rays" (ACRs) once they have been convected out to the solar wind termination shock and accelerated to energies of $\sim 1$ to $100 \mathrm{MeV} /$ nuc. Galactic cosmic rays (GCRs) provide a sample of matter that enters the heliosphere from other regions of the Galaxy.

Figure 2 illustrates typical energy spectra that have been measured for the GCR, ACR, and SEP components at 1 AU. Note that the $\sim 10$ to $\sim 100$ $\mathrm{MeV} /$ nuc energy region covered by SIS includes nuclei originating in each of the three samples of matter to be studied by ACE, with intensities that vary on time scales ranging from minutes to years. The GCR and ACR components, shown at their solar minimum levels, vary by factors of $\sim 10$ and $\sim 100$, respectively, over the solar cycle in this energy range, in inverse correlation with the sunspot number. SEP fluxes, on the other hand, are transient and only rarely reach the intensity levels shown in Figure 2. Figure 3 illustrates the time variation of GCR protons with $>60 \mathrm{MeV}$ over the last two solar cycles. Superimposed on the gradual solar modulation of the GCR component can be seen narrow spikes corresponding to large solar energetic particle events. Based on an extrapolation of the onsets of the last two solar cycles, ACE can expect to observe a marked increase in the frequency of large SEP events just as we enter the next millennium. Up until this onset, SIS will be able to measure the composition of ACRs and GCRs during the waning months of the current solar minimum.

The essential features of SIS are summarized in Table I. The remainder of the paper presents a more detailed discussion of the objectives, the design, and the measured and expected performance of SIS. 


\section{Science Objectives}

\subsection{The IsOTOPIC COMPOSITION OF SOlAR ENERGETIC PARTICLES}

Although the Sun contains the vast majority of solar system material, we have only limited direct knowledge of its isotopic composition. Spectroscopic observations of solar isotopes are very difficult; there are isotope observations for only a few elements and the uncertainties are large. Almost all of the isotopic abundances in the Anders and Grevesse (1989) table of "solar system" abundances are actually based on terrestrial material, while meteoritic measurements (supplemented by spectroscopic data) serve as the standard source for elemental abundances that are used to characterize solar system material.

Solar energetic particles represent a sample of solar material that can be used to make direct measurements of the Sun's elemental and isotopic makeup and to study the most energetic acceleration processes that occur naturally in our solar system. Comprehensive surveys of SEPs have shown that there are two general classes of solar particle events: "gradual" events that are characterized by extended rise times and relatively long durations, and "impulsive" events, generally smaller in size, that are characterized by enhanced fluxes of heavy elements such as $\mathrm{Fe}$, the rare isotope ${ }^{3} \mathrm{He}$, and energetic electrons (see, e.g., Reames, 1995). The ionic charge states in gradual events (e.g., $\mathrm{Fe}^{+15}$ ) are characteristic of coronal temperatures of $\sim 2 \times 10^{6} \mathrm{~K}$ (e.g., Leske et al., 1996b), while those in impulsive events (e.g., $\mathrm{Fe}^{+20}$ ) require a temperature of $\sim 10^{7} \mathrm{~K}$ (Luhn et al., 1987), suggesting that these particles represent the heated flare plasma. SEPs in gradual events are believed to be accelerated by shocks driven by coronal mass ejections (see, e.g., Kahler, 1992).

The compositional differences in these two classes of SEP events are illustrated in Figures 4 and 5. Note that those events with enhanced ${ }^{3} \mathrm{He} /{ }^{4} \mathrm{He}$ ratios all have $\mathrm{Fe} / \mathrm{O}$ ratios greater than the coronal value. The $\mathrm{Fe} / \mathrm{O}$ ratios in events with ${ }^{3} \mathrm{He} /{ }^{4} \mathrm{He}$ ratios $<0.1$ are generally lower, with considerable scatter. Such composition variations are reasonably well organized by the charge to mass ratio $(\mathrm{Q} / \mathrm{M})$ of the ions, and by taking $\mathrm{Q} / \mathrm{M}$-dependent acceleration and transport effects into account it is possible to use SEP measurements in gradual events to obtain coronal abundance measurements for a wide range of elements (Breneman and Stone, 1985).

Earlier SEP studies (see, e.g., Stone et al., 1989) have shown that the elemental composition of the solar corona differs significantly from that of the photosphere in that the abundances of elements with first ionization potential $\geq 10 \mathrm{eV}$ (including, e.g., $\mathrm{He}, \mathrm{N}, \mathrm{O}$, and $\mathrm{Ne}$ ) are depleted by a factor of $\sim 4$ relative to other elements. This difference apparently indicates that neutral species are less efficiently transported from the photosphere to the corona (Meyer, 1985). Although the first measurements of the SEP isotopic composition were made in the late 1970's (e.g., Mewaldt et al., 1984a), such 
measurements have proven to be difficult, and are presently available for only a few elements in a limited number of solar events. Figure 6 summarizes the current state of SEP isotope measurements in large gradual events. In addition, Mason et al. (1994) have presented evidence for an enrichment of heavy isotopes in impulsive solar particle events. The uncertainties in the existing measurements are relatively large as a result of statistical limitations, and in some cases, as a result of background associated with the high count rates in the largest solar events.

With its greatly improved collecting power over earlier instruments, it is hoped that SIS can make a major advance in our knowledge of SEP isotopic composition. Figure 7 shows an estimate of the number of events that SIS would have observed in the gradual SEP event of 10/30/92 measured by SAMPEX (see Selesnick et al., 1993).

\subsection{The Isotopic Composition of ANOMALOUS COSMIC RAYS AND THE LOCAL INTERSTELLAR MEDIUM}

During solar minimum conditions there are seven elements $(\mathrm{H}, \mathrm{He}, \mathrm{C}, \mathrm{N}$, $\mathrm{O}, \mathrm{Ne}$, and $\mathrm{Ar}$ ) whose energy spectra have shown anomalous increases in flux above the quiet time galactic cosmic ray spectrum (see, e.g., the reviews in Klecker, 1995, and Simpson, 1995). This anomalous cosmic ray (ACR) component is now thought to represent neutral interstellar particles that have drifted into the heliosphere, become ionized by the solar wind or ultra-violet radiation, and then been accelerated to energies $>10 \mathrm{MeV} /$ nuc (Fisk et al., 1974), most likely at the solar wind termination shock (Pesses et al., 1981). A unique prediction of this model is that anomalous cosmic rays should be singly ionized, unlike galactic cosmic rays which are essentially fully stripped, and unlike SEPs, which generally have charge states representative of coronal temperatures. Recent measurements from SAMPEX have shown that the bulk of ACR N, O, and Ne with energies $<20 \mathrm{MeV} /$ nuc are indeed singly charged (Klecker et al., 1995), confirming the neutral-gas-origin model. However, some fraction of the ACR ions are ionized further, probably during the acceleration process, and consequently at higher energies most ACRs are multiply charged (Mewaldt et al., 1996). The observed charge states imply that ACRs have passed through less than $1 \mu \mathrm{g} / \mathrm{cm}^{2}$ of material since the time of their acceleration.

Anomalous cosmic ray observations offer a unique opportunity to study a sample of matter from local interstellar space. Because the ACR component apparently represents a direct sample of the local interstellar medium, it carries important information about galactic evolution in the solar neighborhood over the time interval since the formation of the solar nebula some 4.6 billion years ago. This information can be obtained by comparing the isotopic composition

of ACR nuclei with that of solar system abundances, including those measured 
by SIS in solar energetic particles. Radio and optical spectroscopy studies of isotope ratios in interstellar molecules exhibit considerable scatter, but they indicate that isotope abundance ratios such as ${ }^{13} \mathrm{C} /{ }^{12} \mathrm{C},{ }^{15} \mathrm{~N} /{ }^{14} \mathrm{~N}$, and ${ }^{18} \mathrm{O} /{ }^{16} \mathrm{O}$ are dependent on distance from the Galactic center, including variations of as much as a factor of $\sim 2$ from their solar system values (see Figure 8). Variations of this magnitude can be obtained by some galactic evolution models (see, e.g., Audouze, 1983, and Tosi, 1982). Under solar minimum conditions, SIS will observe $\sim 50000$ ACR oxygen, $\sim 8000$ ACR nitrogen, and $\sim 2500$ ACR neon per year. Isotope studies of these elements can lead to improved estimates of the composition of interstellar matter in the vicinity of the Sun.

Recently, there have been new measurements from Wind, Geotail, and Voyager that provide possible evidence for ACR contributions to additional elements, including S, Si, and Fe (see Reames et al., 1997, Takashima et al., 1997, and Stone and Cummings, 1997), with relative abundances $\sim 0.1 \%$ that of oxygen. While these elements are expected to be generally ionized in the interstellar medium, because their first ionization potentials are considerably lower than that of $\mathrm{H}(13.6 \mathrm{eV})$, it is possible that the observed fluxes represent the neutral abundances of these species in the LISM. It is also possible that they originate from other sources of pickup ions (see, e.g., Geiss et al., 1996), since there is as yet no evidence that these enhanced fluxes have ionic charge states of $\mathrm{Q} \leq 4$, as would be expected for ACR nuclei (see Klecker et al., 1997). With its large geometry factor, SIS will be able to search for ACR contributions down to a level of $\sim 10^{-4}$ the intensity of oxygen.

\subsection{Studies of Low ENERgy Galactic Cosmic Ray Isotopes}

Galactic cosmic rays represent an accessible sample of matter that originates outside the solar system. The isotopic composition of this sample of matter contains a record of the nuclear history of cosmic ray material, including its synthesis in stars, and subsequent nuclear interactions with the interstellar gas. The primary instrument for studying galactic cosmic ray isotopes on ACE is the Cosmic Ray Isotope Spectrometer (CRIS) which will cover the energy interval from $\sim 100$ to $500 \mathrm{MeV} /$ nuc with a geometry factor of $>200$ $\mathrm{cm}^{2}$ sr (see Stone et al., 1998b). SIS will extend isotope studies to lower energies with a collecting power that is still several times greater than that of earlier satellite instruments. These measurements will be particularly useful for studying the energy dependence of the isotopic composition.

Neon isotope studies are of particular interest, because there are a variety of different compositions of neon isotopes observed in the solar system (see, e.g., Podosek, 1978). The right-hand panel of Figure 9 summarizes reported measurements of the ${ }^{22} \mathrm{Ne} /{ }^{20} \mathrm{Ne}$ ratio in cosmic rays as a function of energy/nucleon. It is well-known that GCR neon is enriched in ${ }^{22} \mathrm{Ne}$, even when 
corrections are made for contributions from the fragmentation of heavier comic rays during their transport through the interstellar medium. Note that below $\sim 50 \mathrm{MeV} /$ nuc there is a sudden decrease in the ${ }^{22} \mathrm{Ne} /{ }^{20} \mathrm{Ne}$ ratio, as the ACR component begins to dominate. The magnitude of this decrease will, of course, depend on the ${ }^{22} \mathrm{Ne} /{ }^{20} \mathrm{Ne}$ in the LISM, and the curves in Figure 9 illustrate several possibilities.

Figure 9 also summarizes ${ }^{22} \mathrm{Ne} /{ }^{20} \mathrm{Ne}$ measurements from the solar system, galactic cosmic rays, and from the LISM as derived from previous studies of ACRs. The meteoritic and lunar component known as "Neon-B" apparently represents directly implanted solar wind, while "Neon-A" is apparently a primordial component (see, e.g., Podosek, 1978). Galactic cosmic ray source material has an overabundance of ${ }^{22} \mathrm{Ne} /{ }^{20} \mathrm{Ne}$ by a factor of 2.3 to 7 compared to Neon-A and Neon-B. Studies of ACR isotopes to date are limited by statistical uncertainties, but clearly show a ratio much lower than in GCRs (Leske et al., 1997). This implies that GCRs are not simply an accelerated sample of interstellar matter; they must also include contributions from sources that are rich in ${ }^{22} \mathrm{Ne}$, such as Wolf-Rayet stars (Cassé and Paul, 1982; Prantzos et al., 1987). With two years of solar minimum measurements from SIS it should be possible to distinguish whether the $\mathrm{ACR}{ }^{22} \mathrm{Ne} /{ }^{20} \mathrm{Ne}$ ratio agrees with either that of Neon-A or Neon-B.

\section{Instrument Description}

\subsection{APPROACH}

Identification of the charge and mass of energetic nuclei in SIS relies on a refined version of the standard $d E / d x-E$ technique. A particle of charge $Z$, mass $M$, and velocity $v$ has kinetic energy $E$ proportional to $M v^{2}$ and loses energy as it penetrates matter at a rate $d E / d x$ proportional to $Z^{2} / v^{2}$. Taking the product of these two quantities yields

$$
(d E / d x) E \propto Z^{2} M
$$

independent of the particle's velocity. A plot of $d E / d x$ vs. $E$ for a particular nuclide over a range of velocities forms a hyperbola, and the hyperbolas for different nuclides are separated according to their values of $Z^{2} M$.

This approach is illustrated in Figure 10 in which we approximate $d E / d x$ by the ratio $\Delta E / \Delta x$ where $\Delta E$ is the energy deposited by the particle in penetrating a thickness $\Delta x \sim 0.10 \mathrm{~g} / \mathrm{cm}^{2}$ of silicon. The total energy is obtained by adding to $\Delta E$ the energy deposited in a following stack of silicon detectors in which the particle comes to rest. Each point on the plot corresponds to a measured heavy nucleus. The heavy hyperbolic "tracks" corresponding to the elements boron through oxygen are well separated. Each of these 
bands is made up of several more closely spaced tracks corresponding to different isotopes of the element (see inset in Figure 10). The wide spacing of adjacent elements and the closer spacing of successive isotopes results from the quadratic dependence on $Z$ and linear dependence on $M$ in Eq. 1. Typically, the spacing between isotopes is $\sim 1 / 8$ the spacing between elements, so there is no ambiguity in the particle identification as long as the elements of interest have fewer than 8 isotopes present. This is the case for all of the elements to be measured with SIS.

The resolution of the particle identification can be significantly improved by measuring $\Delta E$ in a detector having a thickness, $L$, that is a significant fraction of the particle's range. An equation for the charge and mass of a detected nucleus can be obtained by equating the change in the particle's range in passing through this detector to the thickness of material penetrated. Letting $R_{Z, M}(E / M)$ be the range of a particle of charge $Z$, mass $M$, and kinetic energy $E$ we can write:

$$
R_{Z, M}(E / M)-R_{Z, M}\left(E^{\prime} / M\right)=L \sec (\theta)
$$

where $\theta$ is the angle at which the particle penetrates the $\Delta E$ detector and $E^{\prime}$ is its residual energy when it emerges. Tabulations of the function $R_{Z, M}(E / M)$ are available (e.g., Hubert et al., 1990).

Equation 2 is an implicit expression giving $Z$ and $M$ in terms of the measured quantities $E^{\prime}, \Delta E=E-E^{\prime}, L$, and $\theta$. Solving it typically begins by assuming a mass-to-charge ratio and solving for $Z$. In the resulting charge histogram, different elements are well separated, allowing one to then assign an integer value of $Z$ to each event. With $Z$ known, we then use Equation 2 to solve for $M$. Practical techniques for carrying out these solutions are described in Appendix A.

Using Equation 2, the fundamental equation on which the $\Delta E-E^{\prime}$ technique is based, one can calculate the contributions to the mass resolution from various physical effects such as energy loss fluctuations and multiple scattering and from uncertainties in the measured values of $\Delta E, E^{\prime}, L$, and $\theta$. Additional insight can be obtained using a power-law approximation to the function $R_{Z, M}(E / M)$, which permits an explicit solution for $M$. These techniques are discussed in Stone et al. (1998b).

\subsection{DESIGN REQUIREMENTS}

If an instrument is to achieve the objectives in Section 2 and make a significant contribution beyond previous instruments that have measured solar energetic particles and anomalous cosmic rays, it must satisfy several design requirements.

To resolve adjacent isotopes that differ in abundance by factors of $\sim 100$ requires a mass resolution of $\sim 0.25 \mathrm{amu}$ or better (see, e.g., Stone, 1973). 
For an instrument that uses the multiple $d E / d x-E$ approach, the mass resolution is a sum of several separate contributions (see Appendix A of Stone et al., 1998b) that can be evaluated by taking appropriate partial derivatives of Equation 2 above. Some of these depend on the physics of the interaction of charged particles with matter (e.g., energy-loss fluctuations, multiplescattering, and electron pickup and loss), while others depend on instrument design (e.g., trajectory resolution, uniformity of detector response, and electronic noise levels and stability).

It is also necessary that SIS be capable of returning accurate composition measurements in the presence of high fluxes of low energy protons and helium nuclei. Measurements of solar energetic particles are usually made in a very hostile environment in which the flux of protons $>1 \mathrm{MeV}$ may exceed $10^{5}$ $\mathrm{cm}^{-2} \mathrm{sr}^{-1} \mathrm{sec}^{-1}$. Chance coincidences between these low energy protons and the heavier nuclei that are of primary interest to SIS can potentially lead to ambiguous trajectories or distorted energy loss measurements. To ensure that as few as possible of the nuclei with $\mathrm{Z} \geq 10$ are missed requires that the instrument be capable of selecting the most interesting nuclei for analysis, and that the bit rate be sufficient to transmit several events per second.

Measurements of anomalous cosmic rays, free from contamination of solar and interplanetary particles at lower energy, and free from galactic cosmic rays with higher energies, are best made in the energy interval from $\sim 5$ to $25 \mathrm{MeV} /$ nucleon, where the flux is a decreasing function of energy. Similarly, solar energetic particle spectra typically decrease rather steeply with increasing energy (see Figure 2). It follows that to maximize the number of detected particles for both of these species requires the use of thin detectors with as low a threshold for penetration as possible, combined with large geometry factors. For this reason SIS has two telescopes composed of the largest area Si devices available $\left(\sim 65 \mathrm{~cm}^{2}\right)$. It is also of interest to extend measurements of solar energetic particles to as high an energy as possible in order to understand the acceleration process in these events. The SIS detector stack is composed of devices of graduated thicknesses in order to cover a broad energy range.

The scientific goals described above lead to a number of design requirements on the various subsystems in ACE. For example, the custom VLSI electronics designed for SIS and CRIS are required to have a dynamic range of $>1000$ to accommodate the desired element range, and they need to be both linear and stable over the expected temperature range. Because SIS is designed mainly for heavy ions, the requirements on noise are not as great as they would be for singly-charged particles. The hodoscope for SIS must identify multiple particles with a range of energy losses varying by a factor of $\sim 1000$ and achieve a typical angular resolution of a few tenths of a degree. The thickness of each detector has to be known to an accuracy of $\sim 0.1 \%$. 
The following discussion will document how these design requirements were achieved.

\subsection{THE TELESCOPES}

There are two identical telescopes in SIS, referred to as "A" and "B", respectively. Each is composed of 17 high-purity silicon detectors as shown in Figure 11 and summarized in Table II. The top two detectors, M1 and M2, are position sensitive ("matrix") devices that form the hodoscope and measure both the trajectory and energy loss of incident nuclei. Below M1 and M2 is a 15-element energy-loss stack composed of thicker, single-electrode silicon devices. Stack detectors have $65 \mathrm{~cm}^{2}$ active areas and would be completely circular in shape (with $46 \mathrm{~mm}$ radii) but for a $42 \mathrm{~mm}$ long orientation flat. Thicknesses range from $100 \mu \mathrm{m}$ to $3.75 \mathrm{~mm}$ and progress with depth in each telescope. Note that T6 and T7 are composite devices made by summing the outputs of three and six wafers, respectively. The crystal axes of all detectors are aligned, which allows particle channeling effects to be more easily recognized.

The apertures of the SIS telescopes are covered by three Kapton windows that provide protection from sunlight and micro-meteorites (see Section 3.10). The combined thickness of these windows has a stopping power that is equivalent to $\sim 31 \mu \mathrm{m}$ of silicon.

\subsection{The Matrix Detector Trajectory System}

The matrix detectors are octagonal in shape, 70 to $80 \mu \mathrm{m}$ in thickness, and have $34 \mathrm{~cm}^{2}$ active areas that are divided into 64 strips (see Figure 12). The strips are $0.96 \mathrm{~mm}$ wide, separated by $0.040 \mathrm{~mm}$ gaps, and oriented in perpendicular directions on opposite sides to provide both $\mathrm{X}$ and $\mathrm{Y}$ axis readout. These ion-implanted detectors were produced for SIS by Micron Semiconductor Ltd. using n-type $<111>$ float-zone-refined silicon. The thin silicon wafers are bonded into G-10 mounts with a silicone resin (Shin-etsu KJR-9022E). Redundant sets of aluminum wires are used to connect the aluminum strip contacts on each detector to the corresponding gold-plated copper pads on its mount. Signal lines follow copper traces on a Kapton "flex-strip" to a rigid G-10 connector designed to mate with a high density connector on the board containing the matrix detector pulse-height analyzer (PHA) electronics. A detailed discussion of the design and testing of these devices has been given by Wiedenbeck et al. (1996).

Valid events in SIS typically require a coincidence between M1 A and M2A or between $\mathrm{M} 1 \mathrm{~B}$ and $\mathrm{M} 2 \mathrm{~B}$ (where $\mathrm{A}$ and $\mathrm{B}$ refer to the $\mathrm{A}$ and $\mathrm{B}$ telescopes, respectively). Each of the strips on M1 and M2 is then individually pulseheight analyzed with its own 12-bit analog-to-digital converter (ADC) so that 
the trajectory of heavy ions traversing the system can be separated from the tracks of low energy $\mathrm{H}$ or He that might happen to hit one of these detectors at the same time. The custom VLSI circuitry that accomplishes this is described in detail in Section 3.6.1. Detectors M1 and M2 are separated by $6 \mathrm{~cm}$; the resulting rms angular resolution of the system is $\sim 0.25^{\circ}$, averaged over all angles.

\subsection{ENERGY-LOSS STACK DETECTORS}

The stack detectors were also made by Micron Semiconductor Ltd. using n-type $<111>$ float-zone-refined silicon with ion-implanted electrodes. All detectors were bonded into custom-designed G10 mounts, with electrical connections provided by redundant wires bonded to pads on these mounts. The detectors were screened under bias in a thermally cycled vacuum chamber for many weeks.

Each detector in SIS is biased at least $20 \mathrm{~V}$ beyond its depletion voltage, which in turn was established by scanning alpha particle sources across the ohmic surface while varying the bias and noting when complete charge collection efficiency was obtained. Electronics noise for stack channels T1 to $\mathrm{T} 7$ ranges between 100 and $400 \mathrm{keV}$ rms. Further fabrication, testing, and selection details can be found in Dougherty et al. (1996).

\subsection{ELECTRONICS}

The SIS electronics system is organized as shown in the block diagram in Figure 13. The electronics and packaging design follows closely that of CRIS (see Stone et al., 1998b). Key new features include programmable gate arrays, the card-cage/backplane style packaging using thermally actuated zero insertion force connectors, the modular packaging of silicon detectors onto the same PC boards that carry the front-end electronics, the RTX2010 microprocessor as central controller, and a newly developed pulse-heightanalysis hybrid for stack detector readout. In addition, the SIS matrix detectors are read out using a new CMOS VLSI circuit containing 16 complete pulseheight analyzers on a single chip.

The electronic design provides a high degree of programmability and selective redundancy to increase reliability. Discriminator thresholds and coincidence logic equations may be adjusted to compensate for noisy detectors or other anomalies. High voltage may be commanded off or on separately for each detector element. The A and B telescope front-end electronics are independent and the interface with the spacecraft is redundant. All microprocessor code that is non-critical to communication with the spacecraft can be reprogrammed in-flight. The microprocessor can be booted from any one of four identical copies of the system code. 


\subsubsection{Matrix Detector Electronics}

Matrix detector signals obtained from each of 512 strip electrodes are individually pulse-height analyzed in order to avoid degradation of resolution at the high count rates expected in large solar energetic particle events. The matrix detector pulse-height analyzers were implemented using a custom CMOS VLSI circuit especially developed for SIS. Table III lists the characteristics of this device, and Appendix B discusses the internal design and operation of the circuit. A total of 32 of these 16-channel PHA chips are used in SIS.

\subsubsection{Stack Electronics}

Silicon detector wafers which form the two "stacks" are grouped as discussed earlier to form a total of 16 detector elements, each instrumented with a separate PHA. These analyzers and the associated stack detector modules are mounted on eight nearly identical printed circuit boards ("detector boards"), four in each stack (see Figure 14).

The PHAs are hybrid circuits (see Figure 15) manufactured at Teledyne (Cook et al., 1993b). The only active component in the PHA hybrid is a fully custom bipolar application-specific integrated circuit fabricated at Harris Semiconductor. This new circuit represents an evolution of discrete bipolar transistor designs which were flight proven in numerous space instruments over the past thirty years (see references in Cook et al., 1993a). The PHA hybrid has improved performance relative to the prior design, while using a factor of 10 less board area and a factor of 3 less power.

Each PHA hybrid includes a preamplifier, postamplifier, normally open amplifier/offset gate, and peak detector/Wilkinson ADC. Shaping time constants are $2 \mu \mathrm{sec}$ with a $3 \mu \mathrm{sec}$ peaking time of the bipolar signal. Twelve-bit quantization with a maximum conversion time of $256 \mu \mathrm{sec}$ is obtained with a $16 \mathrm{MHz}$ clock. The PHA hybrid was manufactured in a number of types with preamp feedback and bias configured for different detector capacitances and leakage currents.

PHA hybrid performance exceeds design goals, with typical dynamic range of 2000 , gain stability of $20 \mathrm{ppm} /{ }^{\circ} \mathrm{C}$, offset variation of less than 0.5 channels over $-20^{\circ} \mathrm{C}$ to $+40^{\circ} \mathrm{C}$, and deviation from linearity of less than $0.01 \%$ of full scale over the entire dynamic range. Power consumption is $40 \mathrm{~mW}$. The PHA hybrids do not contain the digital counters and logic needed for the Wilkinson ADCs, which are rather implemented in a nearby ACTEL gate array on the detector board.

Also mounted near the PHA hybrids are test pulsers used to simulate detector signals. There is one test pulser per detector board. The test pulse amplitudes are controlled independently for the A and B stacks by two dual gain 8-bit digital-to-analog converters (DACs). The test pulsers allow thorough verification of the coincidence logic and are used in routine instrument functional checks. 
The amplifier chains in the PHA hybrids are DC coupled from the detector through the postamplifier. This eliminates the need for a long secondary AC coupling time constant and allows the DC level at the postamp output to be used to measure the detector leakage current. Such measurements are routinely incorporated into the instrument housekeeping data and are useful monitors of detector health.

\subsection{ON-BOARD PROCESSING}

The central controller of the SIS instrument is an RTX2010 microprocessor system. The ease of programming and high performance level of this microprocessor allowed simplification of hardware design and testing. The microprocessor controls all instrument subsystems, performs on-board data compression and prioritization, executes commands from the spacecraft, collects engineering data, and formats and transfers the telemetry stream. A unique task of the processor is the management of the matrix detectors. The activities of balancing the individual strip leakage currents and measuring the pedestals (discussed in Appendix B) are carried out by sophisticated routines that have been verified to operate successfully at the high particle rates anticipated during major solar events.

\subsubsection{Coincidence Logic}

Whenever any detector in the SIS instrument is triggered, the coincidence logic decides whether a valid event has occurred. The normal requirement for an event is a coincidence between either M1A and M2A, or between M1B and M2B (see discussion in Section 3.6.1). In the normal mode, the thresholds of M1 and M2 are set at $\sim 4 \mathrm{MeV}$ in order to be insensitive to low-energy protons. However, because $\mathrm{He}$ and other light ions stopping near the back of the detector stacks do not typically deposit more than $4 \mathrm{MeV}$ in M1 and M2, it was necessary to also incorporate additional trigger conditions, as discussed below and summarized in Table IV.

In order to maximize the analysis of the heavy $(Z>3)$ nuclei of interest, it is desirable to rapidly characterize the charge of an incident nucleus. To accomplish this, each of the stack ADCs (T1 to T8) has two programmable discriminator levels. The "medium" levels in each ADC are set just above the maximum energy loss that can be deposited by a stopping proton at the maximum acceptable incidence angle (e.g., $\sim 4 \mathrm{MeV}$ in the $100 \mu \mathrm{m}$ thick T1 detectors) and the "high" levels are set just above the maximum energy loss of a stopping ${ }^{4} \mathrm{He}$. The logic conditions "Mor" (corresponding to the logical "or" of all medium discriminators from T1 to T7) and "Hor" (corresponding to the logical or of all high discriminators from M1 through T7) can then be used to achieve the desired separation, as illustrated in Table IV. 
The large flux of $\mathrm{H}$ and He during large SEP events would cause considerable deadtime if all of these particles were analyzed. On the other hand, it is still of scientific interest to monitor the abundance and energy spectra of these species. To accomplish this, the SIS trigger logic incorporates two programmable timers that can be adjusted from 0 to 130 seconds. The "Hy" timer starts whenever an $\mathrm{H}$ event has been fully analyzed; until this timer expires all events recognized to have $\mathrm{Z}=1$ (on the basis of the Mor and Hor discriminator conditions) will be rapidly rejected. This process takes $\sim 20$ $\mu$ secs, much less than the typical time of $10 \mathrm{msec}$ necessary to fully process an $\mathrm{H}$ event. Once the Hy timer expires, $\mathrm{H}$ analysis is enabled and the logic will again accept the next valid $\mathrm{H}$ event. There is also a "He" timer that serves to throttle the analysis of $\mathrm{He}$ events in the same manner. It is expected that these timers will normally be set at $\sim 10$ seconds; long enough that $\mathrm{H}$ and $\mathrm{He}$ will each account for at most $\sim 1$ percent of analyzed events under high rate conditions.

The "ADC2" trigger mode is used to detect $\mathrm{H}$ and He nuclei that do not trigger the thresholds of M1 and M2 (called M1M and M2M in Table IV). If the $\mathrm{Hy}$ and/or He timers have expired and two consecutive stack ADCs ( 11 through $\mathrm{T} 8$ ) are triggered in coincidence, but not $\mathrm{M} 1 \mathrm{M} \cdot \mathrm{M} 2 \mathrm{M}$, the logic recognizes an $\mathrm{ADC} 2$ condition and initiates $\mathrm{ADC}$ rundown of all stack and matrix ADCs. The microprocessor then polls the resulting energy losses in the matrix detectors and declares a valid event if there are detectable energy losses $\geq 0.5 \mathrm{MeV}$ in at least one $\mathrm{X}$ and one $\mathrm{Y}$ strip in both M1 A and M2A or at least one $\mathrm{X}$ and one $\mathrm{Y}$ strip in both M1B and M2B. If neither of these conditions is met, the logic is reset to wait for the next event. Events recognized by the ADC2 condition are sorted into event buffers in the same manner as M1M-M2M events (see Section 3.7.2). The ADC2 mode can be disabled by command if desired.

There is also an optional analysis mode (ADC3) in which the matrix thresholds are raised to $\sim 16 \mathrm{MeV}$, just beyond the maximum energy loss of an alpha particle, in order to further reduce the deadtime resulting from triggering of M1 and M2 by low energy He. In this mode, light nuclei such as $\mathrm{C}, \mathrm{N}$, and $\mathrm{O}$ that stop near the back of the detector stack do not all trigger M1 and M2; these events are recognized (in a manner analogous to the ADC2 mode) if they trigger three consecutive stack ADCs (ADC3 condition) and also trigger Hor. In this mode the matrix thresholds are lowered to their nominal levels and the coincidence logic reverts back to its nominal state if either of the Hy or He timers has expired.

\subsubsection{Priority System}

During the largest solar particle events there may be up to several hundred $\mathrm{Z} \geq 6$ ions per second triggering SIS. Although SIS is capable of analyzing most of these, the allocated telemetry rate of 1992 bps allows only 10 to 
15 events per second to be stored in the spacecraft solid-state recorder and transmitted to the ground. In order to ensure that the most interesting events are not missed, SIS employs a series of discriminators to identify $\mathrm{H}, \mathrm{He}$, and $\mathrm{Z} \geq 3$ nuclei, and then stores events in a series of 95 prioritized buffers (see Appendix C). The buffers distinguish four element categories $(\mathrm{H}, \mathrm{He}$, $3 \leq \mathrm{Z} \leq 9$, and $\mathrm{Z} \geq 10$ ); range in the instrument (as identified by the last detector triggered); zenith angle $\left(\leq 15^{\circ}, 15^{\circ}\right.$ to $25^{\circ}$, and $\geq 25^{\circ}$; quality of the hodoscope data (number of non-adjacent strips per detector surface); and the "Hazard" flag, which identifies events which occur within $\sim 20 \mu \mathrm{sec}$ of the most recent previous ADC trigger. Each buffer can typically accommodate several events, depending on their length, and includes events from both telescopes.

Events are typically read out of these buffers in order of their priority; however, the first $\mathrm{N}$ events (where $\mathrm{N}$ is a commandable integer) of each 256second major frame are read out by cycling sequentially through the buffers, in order to ensure that all buffers are periodically sampled during high rate periods. Further details of the priority system are discussed in Appendix C.

\subsection{SIS DATA}

Data produced by the SIS instrument consists primarily of "event" data, which records the pulse height and trajectory measurements of each detected nucleus, count rate data, and housekeeping parameters. For each event, the SIS microprocessor reads a 12-bit pulse height from each of the 8 stack-detector PHAs and 256 matrix-detector PHAs in the telescope that was hit. If all this information were to be telemetered to the ground, about 1.5 minor frames ( 1.5 seconds) would be needed to send each event within the 1992 bits per second telemetry rate allocated to SIS. Since many of the pulse heights are typically 0 , especially for the matrix strips, it is possible to compress the data to a much smaller volume. Essentially only the pulse heights in the detectors and strips that were triggered are telemetered, along with their identification. Details of the SIS variable-length, compressed event format are given in Appendix D.

With this event compression scheme, event length varies considerably, ranging from a minimum of 15 bytes for particles stopping in M2 triggering only 4 matrix strips to an extreme maximum of 109 bytes for a pulserstimulated event ("Stim" event) triggering all stack detectors and 31 matrix strips, with the optional extended header (see Appendix D). A "typical" event stopping in T4 with 6 strips (allowing for chance coincidences or noisy strips) is 20 bytes long. After allowing telemetry space for rate and housekeeping data, an average of $~ 10$ events per second may be transmitted, which is better than an order of magnitude improvement over that possible without

using a compressed event format. Along with the "class" selection and buffer prioritization systems described in Appendix C, this innovation allows SIS to 
send a large fraction of the most interesting pulse-height events even during intense solar particle events.

In addition to pulse heights, SIS records and transmits a variety of count rates, some primarily to assess the health of the instrument, others crucial for determining event detection efficiencies in order to properly calculate absolute particle fluxes. These rates are summed over the entire 256 second instrument cycle (SIS does not measure "sector" rates over fractions of the $\sim 12$ second spacecraft spin period and is not designed to look for particle anisotropies). Up to 18 of the 213 total measured rates may be selected by command to be read and transmitted at a higher time resolution of once every 32 seconds.

A number of housekeeping parameters such as temperatures and voltages, as well as indications of the leakage current in each of the stack detectors and matrix strips, are also included in the data. Further information about the rate and housekeeping data and the packaging of all this information into the telemetry format is given in Appendix D.

\subsection{COMMAND SYSTEM}

There are a wide range of parameters and functions of the operation of the SIS instrument that can be modified by command in order to optimize its performance and to preserve as many as possible of the instrument functions in the event of in-flight component failures or degradation. Table $\mathrm{V}$ summarizes these commandable functions. There are a total of sixteen hardware command bits that control the instrument logic for the two telescopes. In addition, there are a wide variety of commandable parameters that include ADC thresholds, detector bias voltages, and in-flight calibration. It is also possible to modify the priority system, the event format, and various software parameters that define the classification and priority of events. If a detector should become noisy, or fail, it is possible to turn off its bias, and/or its ADC.

\subsection{MechaniCAL DeSIGN}

The mechanical design of SIS provides the following features:

- Precise and reproducible mechanical positioning of detectors

- Elimination of coaxial cables

- Close proximity of detectors to their preamplifiers with a minimal number of connection points

- A continuous RF shield around the detectors

- A card cage design for ease of assembly and re-assembly of individual components, and 
- Dry nitrogen purge to detectors until just prior to launch.

These goals have been achieved by utilizing a box design in which individual printed circuit boards (PCBs) slide into card rails and mate with zeroinsertion-force connectors mounted on a PCB backplane at the rear of the box (Figure 16). The card rails then lock the PCBs to the walls of the box using jackscrews accessible from the ends of the card rails after the boards are in place.

Some of the printed circuit boards carry only electronic components, while others include module housings containing multiple detectors (see Figure 14). Each SIS telescope is formed by 6 sets of such detector modules (4 stack detector modules and 2 matrix detector modules), one above the other. The SIS detectors were designed to have their signals and bias voltages carried by flex circuits (printed-circuit traces inside flexible Kapton ribbons; see Figure 12) which is an integral part of each detector and its mount. This approach greatly reduced the number of parts needed for assembly and significantly reduced the cost and time involved in the assembly process. The matrix detector flex circuits plug directly into 2 inch $(5 \mathrm{~cm})$ connectors, two per detector, thereby greatly reducing the amount of room needed to interconnect a detector that has 128 independent connections to preamplifier circuitry. The remaining SIS detectors (the "stack" detectors) use flex circuits which terminate more conventionally through Miraco connectors.

The use of flex circuitry in SIS has generally eliminated the need for coaxial cables between detectors and preamplifiers. Previous telescope designs utilized coaxial cables, but experience has shown that the center conductors are fragile and often break during assembly. Even when coaxial cables are assembled successfully, it has been found that electronic pick-up can be highly dependent on how they are routed. By contrast, the routing of flex circuitry and printed-circuit board traces are under the direct control of the designer. The connector reliability has been found to be generally acceptable.

The isotopic resolution criteria discussed above put limits on acceptable detector-position tolerances. These tolerances are specified in Table VI.

The stack-up of parts was minimized in order to keep the tolerance accumulation to a minimum. Each detector module is surrounded by an iridited aluminum fence which is attached to a ground plane on the upper surface of the corresponding printed circuit board. Feet on the bottom of each such RF shield extend through the printed circuit board and mate to the top of the RF shield on the next module down. This prevents build-up of position errors that might arise from the fact that the multi-layer printed-circuit board thicknesses are relatively difficult to control. After the SIS boards are all installed, but before the card rails are tightened, alignment bolts are inserted from the top of the SIS box, extending from the top plate down through the collimator, through all RF shields except the bottom-most one, and screwed into the 
bottom shield. These bolts are intended primarily to align the modules in a reproducible way. The RF shielding around each module is not perfect, since in general there remains a gap of $0.1 \mathrm{~mm}$ between each shield and the board above it.

SIS uses a compact purging system integrated into the center wall of the instrument which allows the purge to be piped through the RF shielding system and directly into the modules where the detectors reside. Evacuation vents were also designed in for launch. Baffles on the vent ports block light from entering through the instrument walls.

Tests of the matrix detectors subsequent to the initial design showed that the severe acoustic environment during launch has a potential to break the bond wires which connect the matrix detector strips to individual preamplifiers. As a consequence, acoustic doors which can be opened by firing explosive cable cutters were added to the design in order to minimize penetration of acoustic energy into the SIS box via the telescope apertures. At the same time, an additional layer of aluminum, $1.6 \mathrm{~mm}$ thick, was bonded to the exterior walls of the box using a $50 \mu \mathrm{m}$ thick layer of acoustic dampening film. These measures reduced the acoustic levels inside the instrument by about $13 \mathrm{~dB}$ relative to those outside.

The acoustic doors are mounted on the top surface of the SIS box in such a way that they are thermally isolated from the SIS box. Behind each door is a mechanical collimator within which are mounted three Kapton foils. The outermost foil is $25 \mu \mathrm{m}$ thick and has a vacuum deposited aluminum coating (VDA) on the inner surface only. The two inner foils are $8 \mu \mathrm{m}$ Kapton with VDA on both sides. The purpose of the foils is to block sunlight and provide protection from micro-meteorites. Apart from these foils, the field of view of each SIS telescope is completely unobstructed. The outermost foil surface is bare Kapton in order to provide an effective radiator of whatever sunlight is not reflected from the inner VDA surface.

The SIS instrument is mounted on the top deck of the spacecraft on a tilted bracket which points the two telescopes at $25^{\circ}$ to the $+Z$-axis of the spacecraft.

The exterior of SIS, on the sides of the box which face away from the Sun, is covered with silver-coated Teflon to create radiator surfaces. The sides which face the Sun are covered with thermal blanket. An operational heater is mounted internal to SIS, while a survival heater is located under the thermal blanket. The SIS mounting bolts are thermally isolated from SIS via Ultem bushings. The nominal internal operating temperature for SIS is approximately $20^{\circ} \mathrm{C}$. 


\subsection{RESOURCES}

The SIS instrument is housed in a single box measuring $30.0 \times 41.9 \times 27.5$ $\mathrm{cm}$. It has a mass of $21.9 \mathrm{~kg}$, which includes the hardware to attach it to the tilted bracket. The tilted bracket has a mass of $2.0 \mathrm{~kg}$, which includes the hardware which attaches the bracket to the spacecraft. There are two thermal blankets which together have a mass of $0.40 \mathrm{~kg}$.

SIS has a nominal power dissipation of $17.8 \mathrm{~W}$, but the power is activity dependent. During high rate periods the power is expected to rise to $18.3 \mathrm{~W}$. In addition, there are $4.2 \mathrm{~W}$ of operational heaters and $18.2 \mathrm{~W}$ of survival heaters in SIS.

Table I summarizes these and other resource allocations for SIS.

\subsection{EleCtricAl Ground SUPPORT EQUIPMENT}

Electrical ground support equipment was developed to support the testing and calibration of SIS prior to integration on the ACE spacecraft. Hardware interfaces to the instrument (commands, telemetry, power, and housekeeping) were provided by a portable spacecraft simulator unit developed at Johns Hopkins University, Applied Physics Laboratory (JHU/APL). The simulator collects data from the instrument for each minor frame (one per second), formats them, attaches time and other status information, and transmits the resulting data block over an RS232 serial line. It also receives commands over the RS232 connection. Two separate classes of commands to the spacecraft

simulator are provided: those to be passed to the instrument and those to be used for configuring the simulator itself.

A Unix workstation is used to collect the data from the simulator and to send commands to it. A set of software applications was developed to parse the data stream and extract useful quantities from the telemetry packet, to make basic displays for monitoring instrument health and function, and to enable generation of commands and display of command replies. Also, during periods when the instrument was on the spacecraft, socket connections were provided for passing telemetry to the Unix work station for display. These connections were available both on the area net and from remote sites via the Internet. This capability allowed more scientists and engineers to watch instrument performance during critical tests without having to travel to the test site.

Additional software handles the generation of higher-level template-driven displays for on-line monitoring of instrument performance. This software also allows accumulation and live display of histograms and scatter plots of user-selected variables. These displays are particularly useful during particle calibrations of the instrument. 


\section{Calibrations and Performance}

\subsection{CALIBRATIONS OF INDIVIDUAL STACK DETECTORS}

Uncertainties in the detector thickness at a given point introduce corresponding uncertainties in the derived mass. To ensure that this latter error is less than $0.1 \mathrm{amu}$, even for Fe-group nuclei, requires that total and deadlayer thicknesses be known to within $\sim 0.1 \%$ of the total thickness over the entire area of each detector.

Absolute thickness measurements of the SIS detectors were made using mono-energetic beams of ${ }^{36} \mathrm{Ar}$ nuclei at the Michigan State University National Superconducting Cyclotron Laboratory (MSU/NSCL). For the thinner detectors, these beams were sent through nine selected spots on each wafer, and stopped in well-characterized, thick, residual-energy $\left(E^{\prime}\right)$ detectors placed immediately behind. Thicker SIS detectors were raster scanned, producing complete maps of $E^{\prime}$. Care was taken to avoid channeling, and resulting energy-loss uncertainties, by orienting the beam away from crystal axes and planes. To calibrate depth versus $E^{\prime}$, the beam was also sent through "slivers" of silicon left over after orientation flats were cut from other detectors. The thicknesses of these slivers were measured to within $0.12 \mu \mathrm{m}$ using a high-precision mechanical micrometer.

A check on radiation hardness was made when the flux of $870 \mathrm{MeV}^{36} \mathrm{Ar}$ was gradually incremented by factors of 3 until $\sim 2 \times 10^{8}$ particles $/ \mathrm{cm}^{2}$ had been accumulated over an area of $\sim 1 \mathrm{~cm}^{2}$ at approximately the 390 $\mu \mathrm{m}$ depth of one $490 \mu \mathrm{m}$ thick detector. Immediately after the exposure, the leakage current had risen by a factor of $\sim 4$ to $\sim 7 \mu$ A. Subsequently, at room temperature, leakage currents were comparable to those taken before exposure, and no radiation-damage effects were visible in alpha-particle maps of charge collection efficiency.

Maps made using radioactive alpha-particle sources revealed that the deadlayers were uniform to within $\pm 0.05 \mu \mathrm{m}$ on both faces of each detector, with a range from 0.1 to $0.6 \mu \mathrm{m}$ in thickness. The charge collection response near the edge of each detector was also mapped, as were depletion and breakdown characteristics. Devices which performed poorly in any one of these tests were avoided in selecting detectors for the flight telescopes.

\subsection{INTERFEROMETER MAPPING}

The 30 detectors making up the two SIS stacks were selected from a total of more than 60 large area devices with thicknesses ranging from $100 \mu \mathrm{m}$ to $1000 \mu \mathrm{m}$, each of which had to be mapped to determine its thickness profile. Because mapping with accelerator beams of heavy ions requires large amounts of beam time and entails a very extensive data analysis effort, a dual laser interferometer system was developed that is capable of making automated, 
high-precision measurements of detector thickness variations (see description in Milliken et al., 1995).

A comparison of the particle and interferometer approaches to detector mapping is shown in Figure 17.

\subsection{ELECTRONIC CALIBRATIONS}

The electronic stimulus system in SIS has one test pulser associated with each matrix detector strip (512 total) and one test pulser for each stack detector board (four in each telescope). All test pulsers may be pulsed independently, or in coincidence, in any combination. There are two stack detector pulser reference voltages (one for each telescope), and three matrix detector pulser reference voltages (one for the detectors' high-voltage side in each telescope, and one for the detectors' low-voltage side, common to both telescopes). Each reference voltage is generated by an 8-bit DAC with two gain states. The system has the following capabilities: 1) periodically pulse all ADCs with a grid of input levels that will provide relative comparisons of ADC stability and 2) pulse a known pattern of inputs to check whether stimulation events are correctly processed, stored, and read out of the correct buffers. These capabilities were used throughout the pre-flight testing period to monitor the functionality and stability of the instrument electronics, and they provide the basis for in-flight electronic calibrations.

Prior to their installation in the instrument, all of the stack hybrids were individually calibrated with a laboratory test system that included a computercontrolled DAC and calibrated test capacitors. These calibrations were carried out at three temperatures $\left(-20^{\circ} \mathrm{C},+20^{\circ} \mathrm{C}\right.$, and $\left.+40^{\circ} \mathrm{C}\right)$.

\subsection{INSTRUMENT CALIBRATIONS}

In addition to the accelerator calibrations of individual detectors that were described in Section 4.1, an engineering model of SIS was calibrated at the MSU/NSCL in February 1996 with $100 \mathrm{MeV} /$ nuc beams of ${ }^{20} \mathrm{Ne},{ }^{40} \mathrm{Ar}$, and ${ }^{60} \mathrm{Ni}$. In addition, a second, essentially identically instrumented calibration unit, composed of spare detectors and flight electronics, was calibrated at MSU in April, 1997. The SIS flight instrument was calibrated at the Gesellschaft für Schwerionenforschung mbH (GSI) accelerator in Darmstadt, Germany in June, 1996 with beams of $300 \mathrm{MeV} /$ nuc ${ }^{18} \mathrm{O}$ and 300, 500, and $700 \mathrm{MeV} /$ nucleon ${ }^{56} \mathrm{Fe}$.

These calibrations were used to calibrate and check the performance of a variety of instrument functions, including: 1) the response and uniformity of the matrix detector trajectory system, 2) detector thresholds, 3) the coincidence logic, 4) ADC response, 5) counting rates, 6) the priority and readout systems, 7) the variation of detector and telescope response with energy and 
angle, 8) the accuracy of heavy- ion range-energy relations, and 9) the balancing of the matrix strip leakage currents in a high rate environment. In these calibrations the instrument was mounted on a movable stage with two translational and two rotational degrees of freedom so that particles with a variety of angles of incidence could be studied. A system of calibrated absorbers was used to degrade the energy of the beam and cause it to stop at desired ranges within SIS. In addition, a system of polyethylene targets was inserted to cause the primary beam to fragment into lighter species. Figure 18 shows an example of data obtained during one of the fragmentation runs.

\subsection{IN-FLIGHT CALIBRATIONS}

Using the electronic stimulation system described in Section 4.3, it is possible to continually monitor the relative stability of each of the 528 ADCs in SIS. Approximately once per minute the 10 detectors in each of the two telescopes are pulsed at input levels that are gradually incremented through the available dynamic range. These events are flagged and may be used to check ADC performance (pedestals, noise levels, and relative gain) and the instrument logic over the course of time.

SIS will also be calibrated using in-flight particles. The stability of the detectors and electronics will be monitored by observing whether the charge and mass peaks of prominent isotopes such as ${ }^{16} \mathrm{O},{ }^{28} \mathrm{Si}$, and ${ }^{56} \mathrm{Fe}$ vary with time or temperature. It is also planned to check the co-planarity and alignment of individual detectors (relative to the ensemble) by comparing their response to abundant species as a function of zenith and azimuth angles. Such checks might, for example, detect any relative motion of the detectors that occurred as a result of the launch environment. Large solar particle events provide the best in-flight calibration data because they provide excellent statistical accuracy over a short time period.

\subsection{ENERGy RANGe AND COLLECTING POWER}

The energy range of nuclei that can be measured by SIS (Figure 19) is determined by their range-energy relationship: the minimum energy is for nuclei stopping in M2 and corresponds to the thickness of M1 plus the three windows ( $77+31 \mu \mathrm{m}$ of equivalent thickness of silicon), while the maximum energy for nuclei stopping in $\mathrm{T} 7$ (at normal incidence) corresponds to a nominal thickness of $\sim 8.25 \mathrm{~mm}$ of silicon.

At wider angles somewhat higher energy particles stop within the telescope. For Ranges 1 to 7 there are redundant estimates of the charge and mass which provide improved mass resolution. The geometry factor for stopping particles is shown in Figure 20 as a function of energy/nucleon for several abundant species (summed over the two telescopes). 
It is also possible to identify the charge of nuclei that penetrate the entire telescope by comparing their energy losses in the ten devices, M1 to T8. The maximum energy to which these penetrating particles can be identified is expected to be at least twice that of $\mathrm{T} 7$ events and this range is reflected in the "Elements only" band in Figure 19.

The energy range over which SIS can measure $\mathrm{H}$ and $\mathrm{He}$ depends on the exact settings of the matrix detector thresholds (M1M and M2M). For a nominal threshold setting of $\sim 4 \mathrm{MeV},{ }^{3} \mathrm{He}$ and ${ }^{4} \mathrm{He}$ that stop in M2 and T1 will trigger M1M and M2M. For deeper ranges the energy loss in M1 and then $\mathrm{M} 2$ drops below $4 \mathrm{MeV}$, but $\mathrm{H}$ and $\mathrm{He}$ can still be identified using the "ADC2" trigger mode (see Section 3.7 and Table IV) if the energy loss in M1 and $\mathrm{M} 2$ is above the software thresholds M1L and M2L. For M1L $\approx \mathrm{M} 2 \mathrm{~L}$ $\approx 0.5 \mathrm{MeV}$, this mode allows ${ }^{4} \mathrm{He}$ to be identified to the end of $\mathrm{T} 7(\sim 40$ $\mathrm{MeV} / \mathrm{nuc}$ ). If either of these thresholds is raised above their nominal settings, the energy range for He will be decreased.

SIS has much more limited capability to measure protons. For the nominal thresholds discussed above, the proton response extends only from $\sim 6$ to $\sim 11$ $\mathrm{MeV}$. The flux of somewhat higher energy protons can be monitored using count rates from detectors deeper in the telescope (see Section 4.7). SIS does not respond at all to electrons.

\subsection{SiS CONTRIBUtions to THE REAL Time SOlar Wind System}

In June of 1996, just months before the scheduled delivery of the SIS instrument to the spacecraft contractor, NASA Headquarters personnel inquired about the possibility of using ACE instruments to provide real-time measurements of $\sim 10$ to $\sim 100 \mathrm{MeV}$ protons in order to help assess the radiation risk to astronauts on the Space Station during large SEP events. A survey of various instruments on ACE indicated that none had appropriate coincidence count rates for protons $>10 \mathrm{MeV}$ (see Stone et al., 1998a). Although SIS has very limited response to protons that are required to trigger M1 and M2 (see Section 4.6), it was realized that the count rates of detectors deeper in the SIS stack provide a means of monitoring intensity variations of higher energy particles. To implement this approach, the SIS microprocessor was reprogrammed to provide two such rates and the spacecraft real time data system was reprogrammed to read out these rates every 32 seconds and include them in the real-time solar wind (RTSW) data format (see Zwickl et al., 1998).

The two selected rates are T4 singles and a coincidence between T6 and T7. For the nominal thresholds in Table II and for particles incident through the telescope aperture, $\mathrm{T} 4$ responds to $\sim 10$ to $\sim 30 \mathrm{MeV}$ protons, while T6.T7 responds to $\sim 30$ to $\sim 80 \mathrm{MeV}$. There will, of course, be a significant background due to higher energy cosmic rays that pass through the sides of the SIS box, but the minimum required energy is considerably higher in this 
case, and this background should vary slowly over the solar cycle. Most of the response for typical solar particle energy spectra will be through the front of the telescope, and not through the sides. The nominal geometry factor for T4 singles is $\sim 55 \mathrm{~cm}^{2}$ sr (summed over the 2 telescopes), while that for T6.T7 coincidences is $\sim 35 \mathrm{~cm}^{2}$ sr. Data from these two rates are now part of the RTSW data set that also includes data from SWEPAM, MAG, and EPAM (Zwickl et al., 1998). These data are available to NOAA in real time for forecasts of space weather and are also available to the rest of the community through the RTSW website.

\subsection{In-FLIGHT PERFORMANCE}

On August 27, two days after the launch of ACE, SIS was turned on and found to be operating as designed. During the next few months, SIS acquired quiet-time measurements of nuclei with $2 \leq \mathrm{Z} \leq 28$ and was able to identify low-energy enhancements in the energy spectra of the ACR elements $C, N$, $\mathrm{O}, \mathrm{Ne}$, and Ar, as well as reporting the first energy spectra for $\mathrm{ACR}{ }^{22} \mathrm{Ne}$ and ${ }^{18} \mathrm{O}$. These quiet-time data, obtained during the passage of ACE to L1, provided the opportunity to test the various instrument operation modes and to adjust some of the thresholds.

In early November of 1997, there were two large SEP events that provided the first test of the instrument under high-rate conditions. The 11/6/97 event, in particular, provided statistically accurate composition and energy spectra measurements for most of the elements from $\mathrm{C}$ to $\mathrm{Zn}(6 \leq \mathrm{Z} \leq 30)$ up to energies of $\sim 100 \mathrm{MeV} /$ nuc. Preliminary analysis also indicates that there are a number of elements (including $\mathrm{C}, \mathrm{O}, \mathrm{Ne}, \mathrm{Mg}, \mathrm{Si}, \mathrm{S}, \mathrm{Ar}, \mathrm{Ca}, \mathrm{Fe}$, and $\mathrm{Ni}$ ) where more than one isotope is resolved and where it will be possible to make well-resolved measurements of SEP abundance ratios such as ${ }^{13} \mathrm{C} /{ }^{12} \mathrm{C}$, ${ }^{34} \mathrm{~S} /{ }^{32} \mathrm{~S}$, and ${ }^{54} \mathrm{Fe} /{ }^{56} \mathrm{Fe}$.

Although considerable work remains to optimize the mass resolution, preliminary measurements include $\sigma \approx 0.17 \mathrm{amu} \mathrm{rms}$ at $\mathrm{O}$ and $\sigma \approx 0.40$ amu rms at Fe during both quiet time and the large solar events of November, 1997. These preliminary values will undoubtedly improve when additional corrections are applied, but there is presently no indication that the mass resolution degrades during times of peak intensity. In addition, the matrix detector readout approach did prove to be successful in identifying heavy-ion trajectories in those events where a chance coincidence occurred between a heavy ion and a low-energy proton. During the first few months of the mission SIS, was also able to identify several impulsive ${ }^{3} \mathrm{He}$-rich events that were also observed by lower-energy instruments on ACE.

In summary, it appears that ACE was launched at the right time to allow SIS to sample both solar minimum and solar maximum conditions. 


\section{Acknowledgments}

This research was supported by the National Aeronautics and Space Administration at the Space Radiation Laboratory (SRL) of the California Institute of Technology (under contract NAS5-32626 and grant NAGW-1919), the Jet Propulsion Laboratory (JPL), and the Goddard Space Flight Center (GSFC).

We wish to thank the many individuals and organizations who contributed to the development of SIS: J. H. Marshall III of Radcal Corp., J. Gill and B. Mitchell of Harris Corp., and R. McKenzie of Teledyne Corp. played major roles in the design, development, and fabrication of the custom stack VLSI chips and hybrid circuits, while G. Stupian and M. Leung of Aerospace Corp. provided their micro-focus X-ray facility for hybrid screening. S. Kleinfelder helped design the custom matrix VLSI chip which was fabricated by UTMC. C. Wilburn and his staff at Micron Semiconductor Ltd. fabricated the silicon detectors. D. Aalami of Space Instruments was responsible for the design of the power supplies and also contributed to the design, fabrication, and testing of the other electronic assemblies. W. Blanchard of Falcon Services provided all of the board layouts except for the logic and matrix boards, which were provided by J. Stelma of Design Solutions. Electronic assembly was carried out at Caltech and JPL by N. Neverida and T. Ngo-Luu of JPL with the help of T. Dea, who also assisted during conformal coating and environmental testing at JPL. V. Nguyen of Caltech was responsible for most of the subassembly testing. W. Morris of Caltech received and kitted parts and J. Valenzuela of Space Instruments assisted in PCB production and technical document control. B. Williams and J. Krein of JHU/APL were responsible for thermal design and support. L. Stillman of the same institution was responsible for the mechanical design of the door mechanism.

At Caltech, B. Sears, R. Selesnick, J. Cummings, and L. Sollitt provided calibration and analysis support, G. Allbritton was responsible for detector testing, G. Flemming contributed to the flight software, J. Burnham provided engineering support, F. Spalding was responsible for project administration, and D. Kubly and R. Kubly performed a variety of secretarial tasks. In addition, M. Calderon, A. Davis, and T. Garrard assisted with computing issues, R. Paniagua and R. Borup provided support in the Caltech Physics shop, and B. Wong helped with a variety of laboratory tasks. We thank H. Issaian and A. Jefferson for their help with neutron calibrations and R. Kavanaugh for use of the Am-Be neutron source. J. Lopez-Tiana and E. Friese provided purchasing and contract support.

At JPL, J. Rice and C. Cruzan provided assistance with wire-bonding of the detectors, B. Potter assisted in expediting the VLSI build at Harris Corp., D. Cipes-Cwik and R. Hill carried out the hybrid inspections at Teledyne, N. Silva contributed to the assembly of the telescope modules and instrument card cage, as well as development of specifications for magnetics parts, 
W. Powell served as our instrument expediter, K. Evans provided advice on parts, J. DePew provided technical support for calibrations and laboratory testing, and M. Salama and T. Scharton assisted with mechanical design issues along with P. Rentz of EER Systems Corporation. R. Pool consulted on contractual issues.

At GSFC, B. Fridovich provided administrative support, M. Madden and B. Nahory were responsible for detector testing, and S. Hendricks of Swales and Associates, Inc. assisted with mechanical design issues.

Some accelerator testing of the SIS detectors was made possible by N. Anantaraman, R. Ronningen, and the staff of the MSU/NSCL, while H. Specht, D. Schardt, and the staff of the GSI heavy ion accelerator in Darmstadt, Germany made possible the heavy ion calibrations of the SIS instrument. R. Koga provided access to the "Aerospace" chamber at the Lawrence Berkeley Laboratory's 88" Cyclotron where additional detector calibrations and IC latch-up testing took place. The spacecraft team at JHU/APL provided assistance in many areas while SIS was being integrated and tested on the spacecraft.

Finally, we thank A. Frandsen, G. Murphy, H. Eyerly, M. Breslof, C. Rangel, and M. McElveney of the ACE Payload Management Office for their untiring support and assistance, and D. Margolies, J. Laudadio, and the ACE Project Office at GSFC for their help in all phases of the SIS development.

\section{Appendix}

\section{A. Iterative Calculation of Particle Masses}

As discussed in Section 3.1 the fundamental relationship used for deriving a particle's mass from measurements of $\Delta E$, the energy that it loses in traversing a thickness of material $L$, and $E^{\prime}$, the residual energy with which it emerges, is $\mathcal{R}_{Z, M}\left(\left(\Delta E+E^{\prime}\right) / M\right)-\mathcal{R}_{Z, M}\left(E^{\prime} / M\right)=L$ (note: we use $\mathcal{S}_{Z}(E / M)$, $\mathcal{R}_{Z, M}(E / M)$, and $\mathcal{E}_{Z, M}(R)$ to represent the functional dependence of a particle's specific ionization, range, and energy per nucleon on the indicated variables). Using the fact that the particle range is proportional to its mass, this can be written as

$$
\mathcal{R}_{Z, M_{0}}\left(\left(\Delta E+E^{\prime}\right) / M\right)-\mathcal{R}_{Z, M_{0}}\left(E^{\prime} / M\right)=\left(M_{0} / M\right) L,
$$

where $M_{0}$ is the mass of any selected reference isotope of element $Z$.

The solution of Equation A1 for $M$ is complicated by the fact that $M$ appears in the argument of the range function. In fact, this dependence, which is approximately proportional to $M^{-1.7}$, is stronger than the explicit $M^{-1}$ dependence on the right hand side. 
It is useful to develop a formula which isolates most of the $M$ dependence in Equation A1 on one side of an equation so that one can do an iterative calculation of mass which converges in a relatively small number of steps. To obtain such a formula we note that the range-energy relation is reasonably well represented by a power law, $\mathcal{R}_{Z, M}(E / M)=k M / Z^{2}(E / M)^{a}$. Then to a good approximation we can write $\mathcal{R}_{Z, M_{0}}(E / M) \simeq\left(M_{0} / M\right)^{a} \mathcal{R}_{Z, M_{0}}\left(E / M_{0}\right)$. With this substitution Equation $\mathrm{A} 1$ can be solved for $M$ yielding

$$
M \simeq M_{0}\left[\frac{\mathcal{R}_{Z, M_{0}}\left(\left(\Delta E+E^{\prime}\right) / M_{0}\right)-\mathcal{R}_{Z, M_{0}}\left(E^{\prime} / M_{0}\right)}{L}\right]^{1 /(a-1)} .
$$

This equation is used as the basis for an iterative calculation of the particle's mass: replacing $M_{0}$ with an estimate of the mass, the formula yields an improved estimate. Although the value of $a$ has a weak dependence on energy, reasonable convergence is obtained by simply using a constant $a=1.7$.

An additional complication is introduced by the presence of a deadlayer between the layers of material in which $\Delta E$ and $E^{\prime}$ are measured. A particle traversing a deadlayer of thickness $l$ suffers an energy loss $\delta E$ which goes unmeasured. However, we can calculate the expected value of $\delta E$ based on an estimate of the particle's mass. Then if we replace $\Delta E$ in Equation A2 by $\Delta E+\delta E$ and replace $L$ by $L+l$ we obtain a formula which can be applied even when there is a significant deadlayer.

\section{B. Details of the Matrix Detector Electronics}

As discussed in Section 3.6.1, the signals from the four SIS matrix detectors comprise a total of 512 strips, each of which is individually pulse-height analyzed using custom VLSI circuitry developed especially for ACE. A block diagram of one PHA is shown in Figure 21. Each matrix detector strip is DC coupled to the input of a charge-sensitive amplifier (CSA). The output voltage of a precision, unity gain buffer which follows the CSA is used to charge one of three hold capacitors ( $\mathrm{C} 1$ through $\mathrm{C} 3$ ) selected by externally controlled switches. Under quiescent conditions, when no detector pulse is present, the CSA signal is switched between C 2 and C 3 every $3 \mu$ secs. Thus one of these capacitors is charging while the other holds a steady sample of the baseline (which changes slowly over time due to unbalanced DC current integrated by the CSA, see below).

When a heavy nucleus passes through the detector, the ionization charge it produces is integrated by the CSA causing its output to step up (time constant $\sim 100 \mathrm{nsec}$ ) from the present baseline by an amount proportional to the collected charge. Under the control of the external logic, switch SW1 is closed causing capacitor $\mathrm{C} 1$ to charge to the final voltage reached by the 
CSA output. At the same time the alternate sampling of the baseline into $\mathrm{C} 2$ and $\mathrm{C} 3$ is halted and the capacitor with the most recent good sample is connected to the reference input of a comparator. A DC current source causes the voltage on $\mathrm{C} 1$ to linearly ramp down until it reaches the stored baseline voltage. The output of the Wilkinson comparator is then a logic pulse with width proportional to the detected ionization charge. To ensure that the "rundown" time is non-zero even for very small input signals, a constant pedestal voltage is applied via $\mathrm{C} 4$ before beginning the ramp. At the end of the rundown interval the state of a counter running at $8 \mathrm{MHz}$ is latched to give a 12-bit digitized representation of the pulse height.

A second branch of the circuit is used to produce discriminator signals which are used as inputs to external control logic. The CSA output is processed by a source follower and a shaping amplifier with a peaking time of $\sim 500$ nsec. Two discriminators (one of which is shown in Figure 21) compare the shaped pulse with externally provided reference voltages and produce logic pulses for signals above these thresholds. In the operation mode in which SIS is optimized for large SEP events these reference voltages can be set to correspond to the maximum energy loss of a proton in M1 or M2 ( 4 $\mathrm{MeV})$ and the maximum energy loss of an alpha-particle $(\sim 16 \mathrm{MeV})$, and the coincidence logic can then be operated to respond only to $\mathrm{Z}>1$ and/or $\mathrm{Z}>2$ events.

After an event has been processed, an externally-controlled reset switch is closed to discharge the integration capacitor. Because the CSA input is DC coupled to the detector strip, it integrates the strip's DC current as well as the signal charge. The CSA reset switch must be periodically closed to clear this charge from the capacitor. In order to keep this reset interval relatively long (milliseconds) a current-output digital-to-analog converter (IDAC) is provided to add a programmable current to cancel the leakage current to within $\sim 2 \mathrm{nA}$. Under external control the CSA can be allowed to integrate for an extended time $(\sim 500 \mathrm{msec})$ to obtain a precise measurement of the residual unbalanced current. This value is then used as a basis for updating the IDAC setting. In SIS this leakage current balancing is performed every 512 seconds. The IDAC settings are read out as part of the housekeeping data, making it possible to track the leakage current on each strip and monitor the health of the detector. The pedestal values are also monitored by periodically triggering a conversion when no input signal is present.

A CMOS VLSI chip (see Figure 22) was developed which contains 16 of the matrix detector PHA circuits. The processing of signals from the 128 strips on each detector therefore requires a total of eight of these chips. The circuit can be configured to handle either positive or negative charge inputs, so the same types of devices can be used to process signals from both detector surfaces. The flight chips were fabricated using a radiation-hard 1.2 micron process by UTMC. 
For chips containing 16 PHAs a number of signals have been made common to all channels to reduce the complexity of the interfaces to the external circuitry. These include the switch controls for the sample and hold and the CSA reset, the start of the rundown interval, the injection of the pedestal signal, and the triggering of the test pulser. A logical OR is formed of the 16 discriminator outputs to produce a single trigger signal which is brought out for use by external control logic. The OR of the 16 Wilkinson comparator signals is also brought off-chip to indicate when all conversions are complete and to provide a relatively prompt indication of the size of the largest detected signal.

Each PHA has an on-chip test pulser. The analog DC reference for this pulser and the logic pulse used to trigger a pulse are common to all 16 channels. Similarly, all channels share common discriminator reference voltages.

The IDACs used for leakage current compensation are separate for each of the channels, but the digital control inputs for all 16 IDACs are serially loaded through a single input. This serial DC command loop is also used to load other configuration bits, including masks which can be used to remove selected PHAs from the ORs of the trigger and signal comparator signals. This feature is useful for disabling strips which may be generating excessive noise. Another serial connection is used for reading out the digitized pulse heights from all sixteen PHAs.

In order to obtain pulse-height information from these detectors with precision sufficient for isotope identification (few tenths of a percent), it is necessary to correct for strip-to-strip differences of PHA gains, pedestals, and nonlinearities. Furthermore, in large SEP events, it is important to be able to distinguish $\mathrm{H}, \mathrm{He}$, and heavier elements on board so that the limited bit rate available to SIS can be devoted primarily to high-Z, high-energy events, which are of greatest interest. This identification requires on-board pedestal corrections. The pedestals, which are measured every 512 seconds and included in the SIS housekeeping data, are subtracted from each event before it is classified according to charge group. The pedestal-subtracted strip pulse heights are included in event telemetry, but the original pulse heights can be reconstructed if necessary.

Deviations of the matrix PHAs from linearity were measured using a precision pulser prior to installation in the SIS instrument. The linearity is typically excellent (few channels) when the VLSI chips are configured for negative inputs (corresponding to the junction surface of the detector). When used for positive input, the integral nonlinearity is typically a factor of 10 larger, but still correctable.

The relative gains of the 128 PHAs for a given detector were calibrated using heavy ion data. Since a typical particle produces signals corresponding to the same energy deposition for a single strip on each surface of the detector, the ratio of these signals is just the ratio of the gains between the two strips 
(offset corrections having previously been made). A data set from either an accelerator calibration or collected in flight will contain events corresponding to all possible pairs of front/back strips. An iterative procedure can be used to derive all of the strip PHA gains relative to the gain of a selected strip. Power consumption is $13 \mathrm{~mW}$ per strip.

\section{Details of the SIS Priority System}

As discussed in Section 3.7.2, SIS employs a priority system to ensure that the most interesting events are not missed during large solar particle events, when the analyzed event rate will often exceed the telemetry capacity of 10 to 15 events per second. SIS uses a system of discriminators on the ADC outputs of each detector to identify $\mathrm{H}, \mathrm{He}$, and $\mathrm{Z}>3$ nuclei. This information, supplemented by pulse-height and trajectory information from the analyzed events, is used to store events in a series of 95 prioritized buffers listed in Table VII. The buffers are distinguished by range in the instrument, zenith angle, quality of the hodoscope data, and by the "Hazard flag" (see Section 3.7.2). Events are read out of these buffers in order of their priority, with the exception that the first $\mathrm{N}$ events (where $\mathrm{N}$ is a commandable integer) of each 256-second major frame are read out by cycling sequentially through the buffers. The priority of all buffers can be re-assigned by command.

In addition to the priority system discussed above, which is intended to optimize the use of telemetry, an additional system, referred to as the "class" system, is used to prevent the saturation of the on-board computing capability used to select and format events. Before complete read out and processing of the matrix detector data associated with each event (the most computationallyintensive part of event processing), the on-board microprocessor places each event into one of four classes ( $0,1,2$ or 3$)$, based on the approximate charge and range of the event. Events of class 0 include the most desirable high- $Z$ events and are always passed along for complete processing. However, events of class 1,2 , or 3 are discarded if more than 20 events of that class are already awaiting telemetry readout. The class count limit (nominally 20) is commandable separately for each of the three lower priority classes to allow the system to be optimized for SEP count rate environments. In order to allow the proper measurement of event counting rates, the microprocessor maintains counts of the number of events of each class which are examined and of the number in each class which are accepted for complete processing. These counts are included in telemetry every 256 seconds, along with other count rate data. 


\section{SIS Data Formats}

\section{D.1. EVENT DATA}

For each valid particle event, the SIS microprocessor reads a 12-bit pulse height from each of the 8 stack detector PHAs and 256 matrix detector PHAs in the telescope that was hit. To maximize the number of events sent to the ground in the 1992 bits per second telemetry rate allocated to SIS, these event data are compressed into a flexible length format illustrated in Table VIII and described below.

Each normal SIS event starts with a fixed-length, 5-byte header, the first byte of which gives the total length of that event. Also included in the header are tag bits indicating whether or not various optional subfields of data are included in that event, and how many stack pulse heights and matrix strip pulse heights and coordinates are present. Additional information about the event is found in the header, such as whether it was a pulser-produced stimulated event, which event buffer it was assigned to, whether it was from the A or B telescope, and whether any triggers at all were seen in the other telescope, how many matrix strips had non-zero pulse heights, and how long ago (in instrument cycles) the event was put into the event buffer (which is needed to match the event with the appropriate rate sample time). Engineering information may follow in an optional 4-byte extended header, giving the average of the strip PHA offsets from each of the 4 surfaces of the matrix detectors in the hit telescope, as well as the time to peak for the matrix and T1 pulse heights.

Following the header are the pulse heights from the stack detectors, starting from $\mathrm{T} 1$ and continuing through the deepest detector triggered. This consists of a string of 12-bit pulse heights, with an additional 4 bits of zeroes to fill out the last byte if needed.

Matrix detector information is next. The surface number, strip number, and pulse height uses 20 bits for each strip with a non-zero pulse height. By default, information from a maximum of 10 strips on the top of M1, 9 strips on the bottom of M1, 6 strips on the top of M2, and 6 on the bottom of M2 may be telemetered; if more strips are triggered, only those with the largest pulse heights are sent. More strips are allocated by default to M1 than M2 since it is more exposed and should see a higher count rate. These strip allocations may be changed by command to any pattern in which the maximum total is 31 strips.

If the event is pulser-generated, the normal event format concludes with a 10-byte block listing which pulsers were active and the value of their control DAC levels.

In addition to these compressed, normal mode events, the instrument can be commanded to send "diagnostic" mode events. Such an event consists of the compressed event data and raw pulse-height data from all 8 stack PHAs 
and the 256 matrix strips of the triggered telescope, along with tags indicating which discriminators fired and a variety of tag bits revealing the coincidence signals processed by the on-board logic. Such diagnostic events allow potential problems in the onboard compression algorithms to be identified so that they may be fixed by making the appropriate changes to the flight firmware. The raw data part of these events are a fixed 540 bytes in length.

\section{D.2. RATE AND HOUSEKEEPING DATA}

In addition to pulse-height data, SIS records and transmits "singles" count rate data from each of the 16 stack and 4 matrix detectors (useful for monitoring detector health), a variety of different coincidence rates and event processing monitor rates, 3 separate livetimes, and rates from each of the 96 event buffers, which are needed to calculate efficiencies and thus obtain absolute fluxes for each category of events, for a total of 213 different rates. All these rates are accumulated over one 256-second instrument cycle. Any 18 of these 213 rates may be selected by command to be read and transmitted at a higher time resolution of once every 32 seconds. These so-called "high-priority" rates have their appropriate livetime corrections done onboard by the microprocessor. Various housekeeping measurements are also recorded, including voltage monitors for the low voltage power supplies, current monitors for the high voltage supplies, silicon detector leakage currents, and temperatures. Every 512 seconds, the fine and coarse current DAC settings and matrix detector PHA offsets for all 512 matrix strips are sent to the ground. In the remaining telemetry space, the SIS command table is slowly trickled out, taking 15 instrument cycles (64 minutes) to transmit the entire table.

\section{D.3. TELEMETRY FORMAT}

The event, rate, and housekeeping data are packaged into a 249 byte long SIS instrument minor frame. The first bit of this minor frame is used to find the start of the 256-second instrument cycle. It is set to 0 for the first 128 frames, and 1 for the next 128 frames of the cycle. The second bit serves as a flag to indicate that a command response appears in this minor frame, beginning in the seventh byte of the frame. The first byte of a command response gives the length of the remainder of that command echo. The maximum length of a command echo is programmable, but always less than a full minor frame; the rest of the frame after the command response contains normal or diagnostic mode data. All commands are echoed back in the SIS telemetry data, along with any output generated by the command, thus documenting the command history of the instrument within the data stream. The third bit is set if the pulse-height data in this frame is a diagnostic mode event rather than a normal, compressed event. Diagnostic events may span any number 
of contiguous minor frames, and may start in the middle of a normal event (which then continues after the end of the diagnostic event). After these first 3 bits, the remaining 45 bits in the first 6 bytes are allocated to rate and housekeeping data.

Each item of rate or housekeeping data is assigned to one of the 256 minor frames per instrument cycle. These data are packaged to maximize the use of the 45 bits per frame allocated to them. All rates are compressed 15-bit numbers, and are packaged three per frame for those frames in which they appear. Housekeeping data are typically 10-bit quantities packed four per frame, while other frames contain five 8-bit matrix detector PHA offsets or nine 5-bit matrix detector leakage current DAC values. Frames containing part of the command table readout each contain five bytes of the command table. Normal mode pulse-height data, diagnostic event data, or command responses (whichever is indicated by the second and third bits of the frame) fill out the rest of the minor frame after the first 6 bytes.

Events can span the boundary from one frame to the next and are strung sequentially together to maximize the use of telemetry space. Only at the beginning of the 256-second instrument cycle is an event forced to start at the beginning of the event data section of the frame. If one or more frames in the middle of an instrument cycle should be lost due to telemetry problems, it is important to be able to find the starting point of the next event to avoid losing the rest of the pulse-height data from that cycle. This is readily done as follows. Both the total length of the event and the length of each subsection (from which the total length may be independently calculated) are included in fixed positions in the event header (see Table VIII). By assuming that any given byte is the start of an event and comparing its value of the apparent event length with that calculated from the supposed event header, one can test whether the start of an event may indeed have been found. If so, the next byte after its end should also satisfy the criteria to be the start of an event, and so on till the end of the instrument cycle. 


\section{References}

Anders, E. and Ebihara, M.: 1982, 'Solar-system Abundances of the Elements', Geochim. Cosmochim. Acta 46, pp. 2363-2380.

Anders, E. and Grevesse, N.: 1989, 'Abundances of the Elements: Meteoritic and Solar', Geochim. Cosmochim. Acta 53, pp. 197-214.

Audouze, J.: 1983, in:Observational Tests of Stellar Evolution Theory eds. A. Maeder and A. Renzini, IAU Symposium 105, Dordrecht:Reidel, p. 541

Breneman, H. H. and Stone, E. C.: 1985, 'Solar Photospheric and Coronal Abundances From Solar Energetic Particle Measurements', Astrophys. J. Lett. 199, pp. L57-L61.

Cassé, M. and Paul, J. A.: 1982, 'On the Stellar Origin of the Ne-22 Excess in Cosmic-Rays', Astrophys. J. 258, pp. 860-863.

Connell, J. J. and Simpson, J. A.: 1995, 'The Ulysses Cosmic Ray Isotope Experiment II: Source Abundances of $\mathrm{Ne}, \mathrm{Mg}$ and Si Derived from High Resolution Measurements', Proc. 24th Intl. Cosmic Ray Conf. Rome, Italy 2, pp. 602-605.

Cook, W. R., Cummings, A. C., Cummings, J. R., Garrard, T. L., Kecman, B., Mewaldt, R. A., Selesnick, R. S., Stone, E. C. and von Rosenvinge, T. T.: 1993a, 'MAST: A Mass Spectrometer Telescope for Studies of the Isotopic Composition of Solar, Anomalous, and Galactic Cosmic Ray Nuclei', IEEE Trans. Geosci. Remote Sensing 31, pp. 557-564.

Cook, W. R., Cummings, A. C., Kecman, B., Mewaldt, R. A., Aalami, D., Kleinfelder, S. A. and Marshall, J. H.: 1993b, 'Custom Analog VLSI for the Advanced Composition Explorer', Small Instruments Workshop Proc. Pasadena, CA

Cummings, A. C., Stone, E. C. and Webber, W. R.: 1991, 'The Isotopic Composition of Anomalous Cosmic-Ray Neon', Proc. 22nd Intl. Cosmic Ray Conf. Dublin, Ireland 3, pp. 362-365.

Dietrich, W. F. and Simpson, J. A.: 1979, 'The Isotopic and Elemental Abundances of Neon Nuclei Accelerated in Solar Flares', Astrophys. J. Lett. 231, pp. L91-L94.

Dougherty, B. L., Christian, E. R., Cummings, A. C., Leske, R. A., Mewaldt, R. A., Milliken, B. D., von Rosenvinge, T. T. and Wiedenbeck, M. E.: 1996, 'Characterization of Large-Area Silicon Ionization Detectors for the ACE Mission', SPIE Conf. Proc. 2806, pp. 188-198.

Fisk, L. A., Kozlovsky, B. and Ramaty, R.: 1974, 'An Interpretation of the Observed Oxygen and Nitrogen Enhancements in Low-Energy Cosmic Rays', Astrophys. J. Lett. 190, pp. L35L38.

Garrard, T. L. and Stone, E. C.: 1993, 'New SEP-Based Solar Abundances', Proc. 23rd Intl. Cosmic Ray Conf. Calgary, Canada 3, pp. 384-387.

Geiss, J., Gloeckler, G. and von Steiger, R.: 1996, 'Origin of C+ Ions in the Heliosphere', Space Sci. Rev. 78, pp. 43-52.

Geiss, J. F., Buehler, H., Cerutti, H., Eberhardt, P. and Filleux, Ch.: 1972, 'Solar Wind Composition Experiment', Apollo-16 Prelim. Sci. Report, NASA SP-315 231, p. 14-1.

Hubert, F., Bimbot, R. and Gauvin, H.: 1990, 'Range and Stopping-Power Tables for 2.5-500 MeV/Nucleon Heavy Ions In Solids', Atom. Dat. Nucl. Dat. Tables 46, pp. 1-213.

Kahler, S. W.: 1992, 'Solar Flares and Coronal Mass Ejections', Ann. Rev. Astron. Astrophys. 30, pp. 113-141.

Klecker, B.: 1995, 'The Anomalous Component of Cosmic Rays in the 3-D Heliosphere', Space Sci. Rev. 72, pp. 419-430.

Klecker, B., McNab, M. C., Blake, J. B., Hamilton, D. C., Hovestadt, D., Kästle. H., Looper, M. D., Mason, G. M., Mazur, J. E. and Scholer, M.: 1995, 'Charge State of Anomalous Cosmic-Ray Nitrogen, Oxygen, and Neon: SAMPEX Observations', Astrophys. J. Lett. 442, pp. L69-L72.

Klecker, B., Oetliker, M., Blake, J. B., Hovestadt, D., Mason, G. M., Mazur, J. E. and McNab, M. C.: 1997, 'Multiply Charged Anomalous Cosmic Ray N, O, and Ne: Observations With HILT/SAMPEX', Proc. 25th Intl. Cosmic Ray Conf. Durban, South Africa 2, pp. 273-276.

Leske, R. A., Mewaldt, R. A., Cummings, A. C., Cummings, J. R., Stone, E. C. and von Rosenvinge, T. T.: 1996a, 'The Isotopic Composition of Anomalous Cosmic Rays from SAMPEX', Space Sci. Rev. 78, pp. 149-154. 
Leske, R. A., Cummings, J. R., Mewaldt, R. A., Stone, E. C. and von Rosenvinge, T. T.: 1996b, 'Measurements of the Ionic Charge States of Solar Energetic Particles at 15-70 $\mathrm{MeV} /$ nucleon using the Geomagnetic Field', AIP Conference Proc. 374, pp. 41-44.

Leske, R. A., Mewaldt, R. A., Cummings, A. C., Stone, E. C. and von Rosenvinge, T. T.: 1997, 'Updated Measurements of the Isotopic Composition of Interplanetary and Geomagnetically Trapped Anomalous Cosmic Rays', Proc. 25th Intl. Cosmic Ray Conf. Durban, South Africa 2, pp. 321-324.

Luhn, A., Klecker, B., Hovestadt, D. and Möbius, E.: 1987, 'The Mean Ionic Charge State of Silicon in ${ }^{3} \mathrm{He}$-rich Flares', Astrophys. J. 317, pp. 951-955.

Lukasiak, A., Ferrando, P., McDonald, F. B. and Webber, W. R.: 1994, 'Cosmic-Ray Isotopic Composition of C, N, O, Ne, Mg, Si Nuclei in the Energy Range 50-200 MeV per Nucleon Measured by the Voyager Spacecraft During the Solar Minimum Period', Astrophys. $J$. 426, pp. 366-372.

Mason, G. M., Mazur, J. E. and Hamilton, D. C.: 1994, 'Heavy-ion Isotopic Anomalies in ${ }^{3}$ He-rich Solar Particle Events', Astrophys. J. 425, pp. 843-848.

Mewaldt, R. A., Selesnick, R. S., Cummings, J. R., Stone, E. C. and von Rosenvinge, T. T.: 1996, 'Evidence for Multiply-Charged Anomalous Cosmic Rays', Astrophys. J. Lett. 466, pp. L43-L46.

Mewaldt, R. A., Spalding, J. D. and Stone, E. C.: 1984a, 'A High-Resolution Study of the Isotopes of Solar Flare Nuclei', Astrophys. J. 280, pp. 892-901.

Mewaldt, R. A., Spalding, J. D. and Stone, E. C.: 1984b, 'The Isotopic Composition of the Anomalous Low-Energy Cosmic Rays', Astrophys. J. 283, pp. 450-456.

Mewaldt, R. A. and Stone, E. C.: 1989, 'Isotope Abundances of Solar Coronal Material Derived From Solar Energetic Particle Measurements', Astrophys. J. 337, pp. 959-963.

Meyer, J.P.: 1985, 'Solar-Stellar Outer Atmospheres and Energetic Particles, and Galactic Cosmic Rays', Astrophys. J. Suppl. 57, pp. 173-204.

Milliken, B. Leske, R. A. and Wiedenbeck, M. E.: 1995, 'Silicon Detector Studies with an Interferometric Thickness Mapper', Proc. 24th Intl. Cosmic Ray Conf. Rome, Italy 4, pp. $1283-1286$.

Pesses, M. E., Jokipii, J. R. and Eichler, D.: 1981, 'Cosmic Ray Drift, Shock Wave Acceleration, and the Anomalous Component of Cosmic Rays', Astrophys. J. Lett. 246, pp. L85-L89.

Podosek, F.: 1978, 'Isotopic Structures in Solar System Materials', Ann. Rev. Astron. Astrophys. 16, pp. 293-334.

Prantzos, N., Arnould, M. and Arcoragi, J. P.: 1987, 'Neutron-Capture Nucleosynthesis During Core Helium Burning in Massive Stars', Astrophys. J. 315, pp. 209-228.

Reames, D. V.: 1993, 'Mean Element Abundances in Energetic Particles from Impulsive Flares', Proc. 23rd Intl. Cosmic Ray Conf. Calgary, Canada 3, pp. 388-391.

Reames, D. V.: 1995, 'Solar Energetic Particles - A Paradigm Shift', Rev. Geophys. Suppl. 33, pp. 585-589.

Reames, D. V., Barbier, L. M. and von Rosenvinge, T. T.: 1997, 'Wind/Epact Observations of Anomalous Cosmic Rays', Adv. Space Res. 19, pp. 809-812.

Reames, D. V., Cane, H. V. and von Rosenvinge, T. T.: 1990, 'Energetic Particle Abundances in Solar Electron Events', Astrophys. J. 357, pp. 259-270.

Selesnick, R. S., Cummings, A. C., Cummings, J. R., Leske, R. A., Mewaldt, R. A., Stone, E. C. and von Rosenvinge, T. T.: 1993, 'Coronal Abundances of Neon and Magnesium Isotopes from Solar Energetic Particles', Astrophys. J. Lett. 418, pp. L45-L48.

Simpson, J. A.: 1995, 'The Anomalous Nuclear Component in the Three-Dimensional Heliosphere', Adv. Space Res. 16, pp. (9)135-(9)149.

Simpson, J. A., Wefel, J. P. and Zamow, R.: 1983, 'Isotopic and Elemental Composition of Solar Energetic Particles', Proc. 18th Intl. Cosmic Ray Conf. Bangalore, India 10, pp. 322-325.

Stone, E. C.: 1973, 'Cosmic Ray Isotopes', Proc. 13th Intl. Cosmic Ray Conf. Denver 5, pp. 3615-3626.

Stone, E. C., Burlaga, L. F., Cummings, A. C., Feldman W. C., Frain, W. E., Geiss, J., Gloeckler, G., Gold, R., Hovestadt, D., Krimigis, S. M., Mason, G. M., McComas, D., Mewaldt, R. 
A., Simpson, J. A., von Rosenvinge, T. T. and Wiedenbeck, M. E.: 1989, 'The Advanced Composition Explorer', AIP Conference Proc. 203, pp. 48-58.

Stone, E. C., Cohen, C. M. S., Cook, W. R., C Cummings, A. C., Gauld, B., Kecman, B., Leske, R. A., Thayer, M. R., Dougherty, B. L., Grumm, R. L., Milliken, B. D., Radocinski, R. G., Wiedenbeck, M. E., Christian, E. R., Shuman, S., Trexel, H., von Rosenvinge, T. T., Binns, Crary, D. J., W. R., Dowkonnt, P., Epstein, J., Hink, P., Klarmann, J., Lijowski, M. and Olevitch, M.: 1998b, 'The Cosmic Ray Isotope Spectrometer for the Advanced Composition Explorer', Space Sci. Rev., this issue.

Stone, E. C., Frandsen, A. M., Mewaldt, R. A., Christian, E. R., Margolies, D., Ormes, J. F. and Snow, F.: 1998a, 'The Advanced Composition Explorer', Space Sci. Rev., this issue.

Stone, E. C. and Cummings, E. C.: 1997, 'Evidence for Anomalous Cosmic Ray S, Si, and Fe in the Outer Heliosphere and for a Non-ACR Source of S at 1 AU', Proc. 25th Intl. Cosmic Ray Conf. Durban, South Africa 2, pp. 289-292.

Takashima, T., Doke, T., Hayashi, T., Kobayashi, M., Shirai, H., Takehana, N., Ehara, M., Yamada, Y., Yanagita, S., Hasebe, N., Kashiwagi, T., Kato, C., Munakata, K., Kohno, T., Kondoh, K., Murakami, H., Nakamoto, A., Yanagimachi, T., Reames, D. V. and von Rosenvinge, T. T.: 1997, 'The First Observation of Sulfur in Anomalous Cosmic Rays by the Geotail and the Wind Spacecrafts', Astrophys. J. Lett. 477, pp. L111-L113.

Tosi, M.: 1982, 'CNO Isotopes and Galactic Chemical Evolution', Astrophys. J. 254, pp. 699707.

Wiedenbeck, M. E., Christian, E. R., Cook, W. R., Cummings, A. C., Dougherty, B. L., Leske, R. A., Mewaldt, R. A., Stone, E. C., and von Rosenvinge, T. T.: 1996, 'Two-Dimensional Position-Sensitive Silicon Detectors for the ACE Solar Isotope Spectrometer', SPIE Conf. Proc. 2806, pp. 176-187.

Wilson, T. L. and Rood, R. T.: 1994, 'Abundances in the Interstellar Medium', Ann. Rev. Astron. Astrophys. 32, pp. 191-226.

Zwickl, R. D., Sahm, S., Barrett, B., Grubb, R., Detman, T., Raben, V., Smith, C. W., Riley, P., Gold, R., Mewaldt, R. A. and Maruyama, T.: 1998, 'The NOAA Real-Time-Solar-Wind (RTSW) System using ACE Data', Space Sci. Rev., this issue. 
Abbreviations: ACE - Advanced Composition Explorer, ACR - anomalous cosmic ray, ADC - analog-to-digital converter, CRIS - Cosmic Ray Isotope Spectrometer, CSA - chargesensitive amplifier, DAC - digital-to-analog converter, GCR - galactic cosmic ray, GSFC Goddard Space Flight Center, GSI - Gesellschaft für Schwerionenforschung mbH (accelerator laboratory in Darmstadt, Germany), IDAC - current-output digital-to-analog converter, ISEE - International Sun-Earth Explorer, JHU/APL - Johns Hopkins University, Applied Physics Laboratory, JPL - Jet Propulsion Laboratory, LISM - local interstellar medium, MSU Michigan State University, NSCL - National Superconducting Cyclotron Laboratory, PCB printed circuit board, PHA - pulse height analyzer, RTSW - real-time solar wind (data), rms - root mean square, SAMPEX - Solar, Anomalous, and Magnetospheric Particle Explorer, SEP - solar energetic particle, SIS - Solar Isotope Spectrometer, SRL - Space Radiation Laboratory, VDA - vacuum deposited aluminum, VLSI - very large scale integrated (circuit), 
Table I. Summary of SIS Characteristics

\begin{tabular}{|c|c|c|}
\hline Characteristic & Value & Details $^{a}$ \\
\hline Measurement Objective & $\begin{array}{l}\text { Elemental and isotopic composition of solar energetic } \\
\text { particles, anomalous cosmic rays, and low-energy galac- } \\
\text { tic cosmic rays }\end{array}$ & $\S 2$ \\
\hline Measurement Technique & Multiple- $\Delta E$ vs. residual energy plus trajectory & $\S 3.1$, Appendix A, F10 \\
\hline \multicolumn{3}{|l|}{ Sensor System } \\
\hline Energy Loss Measurements & $\begin{array}{l}\text { Two detector stacks, each composed of } 15 \text { ion-implanted } \\
\text { Si detectors of varying thicknesses, all } 10 \mathrm{~cm} \text { in diameter }\end{array}$ & $\S 3.3, \S 4.1, \S 4.2, \mathrm{~T} 2$ \\
\hline Trajectory Measurements & $\begin{array}{l}\text { Two 2-dimensional Si multi-strip detectors per stack; } 6 \\
\mathrm{~cm} \text { separation; position resolution } \approx 0.29 \mathrm{~mm}\end{array}$ & $\S 3.4$ \\
\hline $\begin{array}{l}\text { Charge Interval } \\
\text { Primary Interval } \\
\text { Extended Interval }\end{array}$ & $\begin{array}{l}4 \leq Z \leq 28 \\
1 \leq Z \leq 30\end{array}$ & $\S 4.6, \mathrm{~F} 19$ \\
\hline $\begin{array}{l}\text { Energy Interval for Mass Analysis } \\
\text { O } \\
\mathrm{Si} \\
\mathrm{Fe}\end{array}$ & $\begin{array}{l}10-90 \mathrm{MeV} / \text { nucleon } \\
13-125 \mathrm{MeV} / \text { nucleon } \\
17-170 \mathrm{MeV} / \text { nucleon }\end{array}$ & $\S 4.6, \mathrm{~F} 19, \mathrm{~F} 20$ \\
\hline Field of View & $95^{\circ}$ full angle & F11 \\
\hline Geometrical Factor & $38.4 \mathrm{~cm}^{2} \mathrm{sr}$ & $\S 4.6, \mathrm{~F} 20$ \\
\hline $\begin{array}{l}\text { Event Yields (Solar Minimum) } \\
\text { ACR O } \\
\text { ACR Ne } \\
\text { GCR Si } \\
\text { GCR Fe }\end{array}$ & $\begin{array}{l}50,000 / \mathrm{yr} \\
2,500 / \mathrm{yr} \\
5,000 / \mathrm{yr} \\
5,000 / \mathrm{yr}\end{array}$ & $\S 2.2, \S 4.6$ \\
\hline $\begin{array}{l}\text { Event Yields (Large SEP Event) } \\
\text { O } \\
\mathrm{Fe}\end{array}$ & $>10^{5}$ & $\S 4.6, \mathrm{~F} 7$ \\
\hline $\begin{array}{l}\text { Mass Resolution (rms) } \\
\stackrel{\mathrm{O}}{\mathrm{Fe}}\end{array}$ & $\begin{array}{l}\leq 0.15 \mathrm{amu} \\
\leq 0.35 \mathrm{amu}\end{array}$ & $\S 4.7, \mathrm{~F} 10$ \\
\hline $\begin{array}{l}\text { Resource Allocation } \\
\text { Dimensions }(l \times w \times h) \\
\text { Mass } \\
\text { Instrument Power } \\
\text { Bit Rate }\end{array}$ & $\begin{array}{l}30.0 \times 41.9 \times 27.5 \mathrm{~cm} \\
21.9 \mathrm{~kg} \\
17.8 \mathrm{~W} \\
1992 \mathrm{bits} / \mathrm{sec}\end{array}$ & $\S 3.11$ \\
\hline
\end{tabular}

${ }^{a}$ Sections $(\S)$, Figures $(\mathrm{F})$, and Tables $(\mathrm{T})$ containing additional details 
Table II. SIS Detector and ADC Characteristics

\begin{tabular}{ccccccc}
\hline & Nominal & $\begin{array}{c}\text { Netector } \\
\text { Retector } \\
\text { Thickness } \\
(\mathrm{mm})\end{array}$ & $\begin{array}{c}\text { Active } \\
\text { Area } \\
\left(\mathrm{cm}^{2}\right)\end{array}$ & $\begin{array}{c}\text { Geometry }_{\text {Factor }^{c}} \\
\left(\mathrm{~cm}^{2} \mathrm{sr}\right)\end{array}$ & $\begin{array}{c}\text { Nominal }^{d} \\
\text { ADC } \\
\text { Threshold } \\
(\mathrm{MeV})\end{array}$ & $\begin{array}{c}\text { Nominal } \\
\text { ADC } \\
\text { Full Scale } \\
(\mathrm{MeV})\end{array}$ \\
\hline- & M1 & 0.075 & 34 & $\sim 150$ & $4,0.5^{e}$ & 700 \\
R0 & M2 & 0.075 & 34 & 38.4 & $4,0.5^{e}$ & 700 \\
R1 & T1 & 0.1 & 65 & 38.4 & 1.1 & 900 \\
R2 & T2 & 0.1 & 65 & 37.2 & 1.2 & 900 \\
R3 & T3 & 0.25 & 65 & 36.6 & 1.0 & 1800 \\
R4 & T4 & 0.5 & 65 & 34.4 & 2.2 & 2800 \\
R5 & T5 & 0.75 & 65 & 33.0 & 2 & 4200 \\
R6 & T6 & $2.65^{a}$ & 65 & 27.2 & 5 & 8000 \\
R7 & T7 & $3.75^{b}$ & 65 & 19.4 & 7.5 & 12000 \\
R8 & T8 & 1 & 65 & 19.4 & 1.7 & 540 \\
\hline
\end{tabular}

${ }^{a}$ T6 is composed of three wafers with thicknesses of $1.0,0.9$, and $0.75 \mathrm{~mm}$

${ }^{b}$ Composed of six wafers with thicknesses $1.0,0.75,0.5,0.5,0.5$, and $0.5 \mathrm{~mm}$

${ }^{c}$ Sum of two telescopes; limited to trajectories intersecting the top of $\mathrm{T}(\mathrm{N}+1)$

${ }^{d}$ Thresholds are commandable

${ }^{e}$ Initial trigger requires $4 \mathrm{MeV}$; strips detect $\geq 0.5 \mathrm{MeV}$ (see Section 3.6.2) 
Table III. Matrix VLSI Characteristics

\begin{tabular}{ll}
\hline Parameter & Value \\
\hline PHAs per chip & 16 \\
Power per chip & $13 \mathrm{~mW}$ \\
Full scale signal & $31 \mathrm{pC}(700 \mathrm{MeV} \mathrm{Si})$ \\
Dynamic range (full scale:threshold) & $1400: 1^{a}$ \\
Gain variation & $\leq 10 \%$ \\
Threshold variation & $\sim 10 \%$ rms at $3 \mathrm{MeV}$ \\
ADC type & $12-\mathrm{bit}$ Wilkinson \\
Dead time per event & $(\sim 6+0.125 \mathrm{~N}) \mu \mathrm{sec}^{c}$ \\
Integral nonlinearity (+ input) & $\sim 1 \%$ of full scale \\
Integral nonlinearity $(-$ input $)$ & $\sim 0.1 \%$ of full scale \\
Max. leakage current cancelation & $1 \mu \mathrm{A}$ \\
Package size & $(13.5 \mathrm{~mm})^{2}, 84$ pins \\
Temperature coeff. of gain & $< \pm 20 \mathrm{ppm} /{ }^{\circ} \mathrm{C}$ \\
Offset temp. variation $\left(-20^{\circ} \mathrm{C}\right.$ to $\left.40^{\circ} \mathrm{C}\right)$ & $<1 \mathrm{chan}($ after correction) \\
Cross talk for full scale pulse & 2 chan \\
Radiation tolerance & $\sim 100 \mathrm{kRads}$ \\
\hline
\end{tabular}

${ }^{a}$ Noise $\leq 100 \mathrm{keV}$ rms, excluding quantization error.

${ }^{b}$ Among channels on one chip. Chip-to-chip variations can be larger.

${ }^{c} N=$ pulse-height channel number, including pedestal. 
Table IV. SIS Coincidence Requirements

\begin{tabular}{ccccc}
\hline Species & Equation & Hy-Enable & He-Enable & $\begin{array}{c}\text { Matrix } \\
\text { Threshold } \\
\text { State }\end{array}$ \\
\hline $\mathrm{Z} \geq 3$ & $\mathrm{M} 1 \mathrm{M} \cdot \mathrm{M} 2 \mathrm{M} \cdot \mathrm{Hor}$ & - & - & Low \\
$\mathrm{Z} \geq 3$ & $\mathrm{M} 1 \mathrm{H} \cdot \mathrm{M} 2 \mathrm{H} \cdot \mathrm{Hor}$ & False & False & High \\
$\mathrm{Z} \geq 3$ & $\mathrm{M} 1 \mathrm{~L} \cdot \mathrm{M} 2 \mathrm{~L} \cdot \mathrm{Hor} \cdot \mathrm{ADC} 3$ & False & False & High \\
$\mathrm{Z}=2$ & $\mathrm{M} 1 \mathrm{M} \cdot \mathrm{M} 2 \mathrm{M} \cdot \mathrm{Mor} \cdot \overline{\mathrm{Hor}}$ & - & True & - \\
$\mathrm{Z}=2$ & $\mathrm{M} 1 \mathrm{~L} \cdot \mathrm{M} 2 \mathrm{~L} \cdot \mathrm{Mor} \cdot \overline{\mathrm{Hor}} \cdot \mathrm{ADC} 2$ & - & True & - \\
$\mathrm{Z}=1$ & $\mathrm{M} 1 \mathrm{~L} \cdot \mathrm{M} 2 \mathrm{~L} \cdot \overline{\mathrm{Mor}} \cdot \mathrm{ADC} 2$ & True & - & - \\
\hline
\end{tabular}

Matrix Threshold State $=$ commandable two-state $($ High/Low $)$ flag

$\mathrm{M} 1 \mathrm{H}=$ commandable discriminator threshold set nominally at $\sim 16 \mathrm{MeV}$ in M1

$\mathrm{M} 2 \mathrm{H}=$ commandable discriminator threshold set nominally at $\sim 16 \mathrm{MeV}$ in M2

$\mathrm{M} 1 \mathrm{M}=$ commandable discriminator threshold set nominally at $\sim 4 \mathrm{MeV}$ in M1

$\mathrm{M} 2 \mathrm{M}=$ commandable discriminator threshold set nominally at $\sim 4 \mathrm{MeV}$ in M2

$\mathrm{M} 1 \mathrm{~L}=$ energy loss $\geq 0.5 \mathrm{MeV}$ in M1, software threshold

$\mathrm{M} 2 \mathrm{~L}=$ energy loss $\geq 0.5 \mathrm{MeV}$ in $\mathrm{M} 2$, software threshold

Mor = logical "or" of all $\mathrm{M}(\mathrm{Z} \geq 2)$ thresholds

Hor = logical "or" of all $\mathrm{H}(\mathrm{Z} \geq 3)$ thresholds

ADC $2=$ coincidence between two consecutive stack ADCs

ADC3 = coincidence between three consecutive stack ADCs

Hy-Enable $=$ True only after expiration of Hy-timer

He-Enable $=$ True only after expiration of He-timer

$-=$ Not Applicable 
Table V. SIS Command Summary

\begin{tabular}{ll}
\hline Item & Comments \\
\hline Coincidence Logic & \\
Coincidence Equation & Include any/all terms of M1·M2·T1·T2 \\
Hazard Event & Allow/disallow analysis of hazard events \\
Stim Events & Give Stim events highest priority \\
ADC2 Definition & Enable/disable front/back halves of telescope \\
ADC3 Definition & Enable/disable front/back halves of telescope \\
Instrument Operation & \\
Adjust ADC Thresholds & Raise/lower in $10 \%$ steps \\
ADC operation & Enable/disable each individually \\
Matrix strip operation & Enable/disable \\
Matrix strip thresholds & Raise/lower in $10 \%$ steps \\
Medium and High ADC thresholds & Raise/lower in $10 \%$ steps \\
Hy and He event timers & Adjust from 0 to 130 sec \\
Stack detector bias voltages & Raise/lower in $20 \%$ steps \\
Matrix detector bias voltages & Raise/lower in $2 \%$ steps \\
Software Parameters & \\
Angle and Charge Buffers & Adjust boundaries \\
Readout Priority system & Re-define event buffer readout priorities \\
Number of strips selected per matrix layer & Adjust max and select values \\
Event format & Include extended header \\
High priority rates & Select which rates are included \\
Stim events & Change frequency and masks \\
Diagnostic events & Change frequency \\
\hline & \\
\hline &
\end{tabular}


Table VI. SIS Tolerances

\begin{tabular}{lll}
\hline Lateral position tolerance & Matrix: & $0.1 \mathrm{~mm}$ \\
& Stack: & $1.0 \mathrm{~mm}$ \\
Detector co-planarity & Matrix: & $0.1^{\circ}$ \\
& Stack: & $0.1^{\circ}$ \\
Detector azimuthal orientation & Matrix: & $0.1^{\circ}$ \\
& Stack: & $1.0^{\circ}$ \\
\hline
\end{tabular}

pap_revised_9.tex; 15/07/1998; 8:50; no v.; p.42 
Table VII. SIS Event Buffers and Priorities

\begin{tabular}{|c|c|c|c|c|c|c|c|c|c|}
\hline Charge & Range & $0-15^{\circ}$ & $\begin{array}{c}\mathrm{mh}=0 \\
\mathrm{hz}=0 \\
15-25^{\circ}\end{array}$ & $\geq 25^{\circ}$ & $0-15^{\circ}$ & $\begin{array}{c}\mathrm{mh}=0 \\
\mathrm{hz}=1 \\
15-25^{\circ}\end{array}$ & $\geq 25^{\circ}$ & $\begin{array}{r}\mathrm{mh}=1 \\
\mathrm{hz}=0 \\
0-50^{\circ}\end{array}$ & $\begin{array}{c}\mathrm{mh}=1 \\
\mathrm{hz}=1 \\
0-50^{\circ}\end{array}$ \\
\hline \multirow[t]{5}{*}{$\mathrm{Z} \geq 10$} & 8 & \multicolumn{3}{|c|}{62} & \multicolumn{3}{|c|}{61} & \multicolumn{2}{|c|}{60} \\
\hline & $5,6,7$ & 126 & 125 & 116 & 124 & 123 & 115 & 118 & 117 \\
\hline & 3,4 & 122 & 121 & 112 & 120 & 119 & 111 & 114 & 113 \\
\hline & 1,2 & 94 & 93 & 74 & 92 & 91 & 72 & 86 & 84 \\
\hline & 0 & 90 & 88 & 73 & 89 & 87 & 71 & 85 & 83 \\
\hline \multirow[t]{5}{*}{$3 \leq \mathrm{Z} \leq 9$} & 8 & \multicolumn{3}{|c|}{62} & \multicolumn{3}{|c|}{61} & \multicolumn{2}{|c|}{60} \\
\hline & $5,6,7$ & 110 & 109 & 106 & 104 & 103 & 102 & 98 & 97 \\
\hline & 3,4 & 108 & 107 & 105 & 101 & 100 & 99 & 96 & 95 \\
\hline & 1,2 & 82 & 81 & 70 & 79 & 78 & 69 & 66 & 64 \\
\hline & 0 & 80 & 76 & 68 & 77 & 75 & 67 & 65 & 63 \\
\hline \multirow[t]{5}{*}{$\mathrm{Z}=2$} & 8 & \multicolumn{3}{|c|}{45} & \multicolumn{3}{|c|}{44} & \multicolumn{2}{|c|}{34} \\
\hline & $5,6,7$ & \multicolumn{3}{|c|}{59} & \multicolumn{3}{|c|}{54} & \multicolumn{2}{|c|}{41} \\
\hline & 3,4 & \multicolumn{3}{|c|}{58} & \multicolumn{3}{|c|}{53} & \multicolumn{2}{|c|}{40} \\
\hline & 1,2 & \multicolumn{3}{|c|}{57} & \multicolumn{3}{|c|}{52} & \multicolumn{2}{|c|}{39} \\
\hline & 0 & \multicolumn{3}{|c|}{49} & \multicolumn{3}{|c|}{48} & \multicolumn{2}{|c|}{38} \\
\hline \multirow[t]{4}{*}{$\mathrm{Z}=1$} & 8 & \multicolumn{3}{|c|}{43} & \multicolumn{3}{|c|}{42} & \multicolumn{2}{|c|}{33} \\
\hline & $5,6,7$ & \multicolumn{3}{|c|}{56} & \multicolumn{3}{|c|}{51} & \multicolumn{2}{|c|}{36} \\
\hline & 3,4 & \multicolumn{3}{|c|}{55} & \multicolumn{3}{|c|}{50} & \multicolumn{2}{|c|}{35} \\
\hline & 2 & & 47 & & & 46 & & & \\
\hline Stim & & & & & & & & & \\
\hline
\end{tabular}

Notes:

Buffer numbers correspond to readout priorities; highest number $=$ highest priority (buffer numbers 0-32 are unassigned spares)

"Range" refers to the last detector in which an energy loss is measured, i.e., Range 3 means no signal is recorded from beyond T3

"mh" (multi-hodoscope) events have two non-adjacent matrix strips in one or more layers

"hz" (hazard) events occur within $20 \mu \mathrm{sec}$ of a previous ADC trigger 
Table VIII. SIS Normal Event Format

\begin{tabular}{ccccl} 
Item \# & Count & Bits & Sum & Description \\
\hline \multicolumn{7}{l}{ Event Header (fixed length): } \\
\hline 0 & 1 & 1 & 1 & Matrix detector not triggered \\
1 & 1 & 7 & 8 & Number of bytes in this event \\
2 & 1 & 1 & 9 & Stimulated event flag \\
3 & 1 & 7 & 16 & Event buffer ID \\
4 & 1 & 1 & 17 & Primary telescope $(\mathrm{A}=0$; B=1) \\
5 & 1 & 1 & 18 & Other telescope also hit? \\
6 & 1 & 1 & 19 & Include time to peak \& average matrix offsets data block? \\
7 & 1 & 1 & 20 & Hazard flag \\
8 & 1 & 4 & 24 & Range ( $R 1)$ in primary telescope \\
9 & 1 & 3 & 27 & \# Other matrix groups not transmitted (encoded) \\
10 & 1 & 5 & 32 & \# Matrix strips ( $N 1)$ in primary telescope \\
11 & 1 & 3 & 35 & "Time tag" - 3 LSBs of major frame counter (256 s) \\
12 & 1 & 5 & 40 & "Phase"
\end{tabular}

Time to Peak \& Matrix Offset Data (if item \#6 true; fixed length):

\begin{tabular}{lllll}
\hline 13 & 1 & 4 & 4 & Time to peak for T1 pulse height, in units of $0.25 \mu \mathrm{s}$ \\
14 & 1 & 4 & 8 & Time to peak for M2 pulse height, in units of $0.25 \mu \mathrm{s}$ \\
15 & 1 & 4 & 12 & Time to peak for M1 pulse height, in units of $0.25 \mu \mathrm{s}$ \\
16 & 1 & 5 & 17 & Average of M1G strip offsets \\
17 & 1 & 5 & 22 & Average of M1H strip offsets \\
18 & 1 & 5 & 27 & Average of M2G strip offsets \\
19 & 1 & 5 & 32 & Average of M2H strip offsets
\end{tabular}

Stack (variable length):

\begin{tabular}{|c|c|c|c|c|}
\hline 20 & $R 1$ & 12 & 0 to 96 & Stack detector pulse heights +4 bits 0 pad if $R 1$ is odd \\
\hline \multicolumn{5}{|c|}{ Matrix (variable length): } \\
\hline \multirow[t]{9}{*}{21} & $N 1$ & 2 & 2 & Plane number \\
\hline & & 6 & 8 & Strip number \\
\hline & & 12 & 20 & Strip pulse height \\
\hline & & . & . & . \\
\hline & & . & . & . \\
\hline & & . & . & . \\
\hline & & & $\leq 620$ & +4 bits of 0 pad if $N 1$ is odd \\
\hline & & & & (Defaults of $10,9,6$, and 6 strips/plane respectively; \\
\hline & & & & commandable to max total of 31 strips) \\
\hline \multicolumn{5}{|c|}{ Stimulated Event Parameters (if item \#2 true; fixed length): } \\
\hline 22 & 1 & 8 & 8 & Stim mask - flags for which pulsers are active, MSB \\
\hline 23 & 1 & 8 & 16 & Stim mask - flags for which pulsers are active, LSB \\
\hline 24 & 1 & 8 & 24 & VREF1 - tel. A stack DAC level \\
\hline 25 & 1 & 8 & 32 & VREF2 - tel. B stack DAC level \\
\hline 26 & 1 & 8 & 40 & Matrix ground side VREF \\
\hline 27 & 1 & 8 & 48 & Matrix HV side VREF, tel. A \\
\hline 28 & 1 & 8 & 56 & Matrix HV side VREF, tel. B \\
\hline 29 & 1 & 2 & 58 & spare \\
\hline 30 & 1 & 1 & 59 & GAIN_F2 \\
\hline 31 & 1 & 1 & 60 & GAIN_F1 \\
\hline 32 & 1 & 4 & 64 & VREF1 and VREF2 gain bits \\
\hline 33 & 1 & 8 & 72 & Cal sequence $\mathrm{MSB}$ \\
\hline 34 & 1 & 8 & 80 & Cal sequence LSB \\
\hline
\end{tabular}




\section{Figure Captions}

Figure 1. Photograph of the SIS instrument with the acoustic covers open. The dimensions of the housing are $30.0 \times 41.9 \times 27.5$ $\mathrm{cm}$.

Figure 2. Solar-minimum energy spectra measured for ACR and GCR carbon and oxygen on IMP-8 are compared with the oxygen spectrum measured during the large SEP event of 9/23/78 by ISEE-3 (Mewaldt et al., 1984a, 1984b). Curves have been drawn through the data points to guide the eye. Note that during the SEP event, solar particles dominate the observed flux below $\sim 100 \mathrm{MeV} /$ nuc.

Figure 3. Measured flux of protons $>60 \mathrm{MeV}$ from 1973 to 1996 measured by the CPME experiment on IMP-8 (T. Armstrong, private communication). The steady flux varying from $\sim 0.2$ $\mathrm{cm}^{-2} \mathrm{sr}^{-1} \mathrm{~s}^{-1}$ at solar max to $\sim 0.4 \mathrm{~cm}^{-2} \mathrm{sr}^{-1} \mathrm{~s}^{-1}$ is due primarily to galactic cosmic rays. The superimposed spikes (typically several days wide) represent solar proton events. Note that the solar cycle dependence of these two components is anticorrelated.

Figure 4. Characteristics of solar energetic particle events. The left hand panel shows the intensity of 1.9-2.8 MeV/amu Fe versus the intensity of $\mathrm{O}$ at the same energy for 90 different electron events (reproduced from Reames et al., 1990). ${ }^{3} \mathrm{He}-$ rich events are indicated by the circle size, which increases logarithmically with ${ }^{3} \mathrm{He} /{ }^{4} \mathrm{He}$ as shown. The right hand panel shows the histogram of $\mathrm{Fe} / \mathrm{O}$ for the same electron events, with the ${ }^{3} \mathrm{He}$-rich subset blackened. The diagonal line in the left hand panel is drawn at the "mass-unbiased" coronal abundance of Meyer (1985) and the same value is indicated in the right hand panel. Note that the largest ${ }^{3} \mathrm{He}$-rich events are generally 1 to 3 orders of magnitude smaller in size than the largest gradual events.

Figure 5. Average composition of gradual and impulsive SEP events (from Reames, 1993) normalized to coronal abundances tabulated by Garrard and Stone (1993). 
Figure 6. Summary of published measurements of isotope ratios in gradual SEP events for the elements $\mathrm{C}, \mathrm{N}, \mathrm{O}, \mathrm{Ne}$, and $\mathrm{Mg}$, based on a similar figure from Selesnick et al. (1993). Shown are measurements from SAMPEX (Selesnick et al., 1993), ISEE-3 (Mewaldt et al., 1984a; Mewaldt and Stone, 1989), Phoenix-I/S81-1 (Simpson et al., 1983), and IMP-8 (Dietrich and Simpson, 1979). Also shown are solar wind measurements from Geiss et al. (1972) and solar system abundances from Anders and Ebihara (1982).

Figure 7. Estimated number of events of each isotope that SIS would have observed in the large, gradual SEP event of 10/30/92, based on composition measurements from SAMPEX (D. Williams, private communication). The abundances of the isotopes within each element (connected by a solid line) are assumed to be the same as in Anders and Grevesse (1989).

Figure 8. Survey of isotope measurements as a function of distance from the Galactic center (based on data in Figure 2 of Wilson and Rood, 1994). Data for the ${ }^{13} \mathrm{C} /{ }^{12} \mathrm{C}$ ratio are based on radio astronomy measurements of $\mathrm{CO}$ (open circle) and $\mathrm{H}_{2} \mathrm{CO}$ (plus). The ${ }^{15} \mathrm{~N} /{ }^{14} \mathrm{~N}$ data are based on measurements of $\mathrm{HCN}$ (open circle) and $\mathrm{NH}_{3}$ (plus), and the ${ }^{18} \mathrm{O} /{ }^{16} \mathrm{O}$ data are based on $\mathrm{H}_{2} \mathrm{CO}$. The Galactic center data are shown as filled squares, and the solar system values from Anders and Grevesse (1989) are shown as filled circles at $8.5 \mathrm{kpc}$.

Figure 9. Summary of measurements of the ${ }^{22} \mathrm{Ne} /{ }^{20} \mathrm{Ne}$ ratio (adapted from Leske et al., 1996a). On the right are shown measurements of cosmic rays. When the GCR ratio of $\sim 0.6$ (assumed to be valid $\geq 50 \mathrm{MeV} / \mathrm{nuc}$ ) is corrected for contributions from cosmic ray secondaries it results in a cosmic ray source ratio of $\sim 0.27$ to $\sim 0.5$ when cross section and other uncertainties are included. Below $\sim 40 \mathrm{MeV} / \mathrm{nuc}$ ACRs dominate and the measured ratio decreases; the curves show the ratio expected for various assumed abundances in the local interstellar medium. On the left are summarized lunar and meteoritic components (Neon-A and NeonB; see, e.g. Podosek, 1978); the solar wind value from Apollo (Geiss et al., 1972), SEP measurements from SAMPEX (Selesnick et al., 1993), the GCR source value spanning the measurements from Ulysses (Connell and Simp- 
son, 1993) and Voyager (Lukasiak et al., 1994), and the average of the LISM value based on ACR isotope measurements from Voyager and SAMPEX (Cummings et al., 1991; Leske et al., 1996a).

Figure 10. Illustration of the $d E / d x-E$ technique using data acquired during an accelerator calibration of SIS at GSI in Darmstadt, Germany.

Figure 11. Scale drawing of one of the two SIS telescopes, showing only the active regions of the detectors. Detector designations are listed at right, while the total silicon thicknesses are listed at left. The metallized kapton entrance foils total $\sim 7.2 \mathrm{mg} / \mathrm{cm}^{2}$ thick. The opening angle of $95^{\circ}$ is an average which accounts for the octagonal shape of the matrix detectors.

Figure 12. Schematic of a SIS matrix detector in its mount. Each surface of the detector has 64 metallized strips. The X-surface strips are orthogonal to the Y-surface strips and all strips are individually pulse-height analyzed.

Figure 13. Functional block diagram for the SIS electronics.

Figure 14. Photograph of a SIS stack detector module mounted in its board. There are two or more detectors mounted in each module on each stack board (e.g., T1 and T2).

Figure 15. Photograph of a SIS stack hybrid showing the custom bipolar VLSI chip developed for ACE surrounded by passive feedback and filtering components.

Figure 16. Cut-away view of SIS, showing one of the two SIS telescopes. At the top of the cut-away section, one can see three entrance foils supported by the entrance collimator. Below are two matrix detectors followed by the four modules which hold $\mathrm{T} 1+\mathrm{T} 2, \mathrm{~T} 3+\mathrm{T} 4, \mathrm{~T} 5+\mathrm{T} 6$, and $\mathrm{T} 7+\mathrm{T} 8$. The modules are cut away to show the detectors. The power supply appears below the telescope. The acoustic doors, shown in Figure 1, are not included here. 
Figure 17. Comparison of particle (dotted) and interferometer (solid) maps of one $750 \mu \mathrm{m}$ thick stack detector. The contours are labeled for absolute thickness in units of microns. The particle map was produced using residual-energy measurements from a beam of penetrating ${ }^{36} \mathrm{Ar}$ nuclei that was raster-scanned across the detector. Statistical fluctuations are indicated by the raggedness of this map. The interferometry used two coaxial laser beams simultaneously reflecting off the mirrored surfaces of the detector sides.

Figure 18. Scatter plot of $\Delta E$ vs. $E^{\prime}$ for events collected in a SIS accelerator calibration run at the GSI accelerator in Darmstadt, Germany. The data that are plotted were collected for heavy nuclei that stopped in T7 (used as $E^{\prime}$ ) and the sum of the signals from T1 through T6 is used as $\Delta E$. In this run a primary beam of ${ }^{56} \mathrm{Fe}$ at $500 \mathrm{MeV} /$ nucleon was allowed to fragment in a target. Data are shown for elements near $Z=20$. Tracks corresponding to a number of isotopes of each element are clearly visible in these data.

Figure 19. SIS energy range for nuclei with $1 \leq \mathrm{Z} \leq 30$. In addition, SIS will make exploratory measurements of SEP nuclei with $31 \leq \mathrm{Z} \leq 40$.

Figure 20. Geometry factor versus energy for the two SIS telescopes combined, where events passing out the side of the telescopes are eliminated.

Figure 21. Block diagram of one matrix PHA.

Figure 22. Photograph of a matrix VLSI chip (packaged). Each chip contains 16 complete ADC channels and dissipates 13 $\mathrm{mW} /$ channel. 


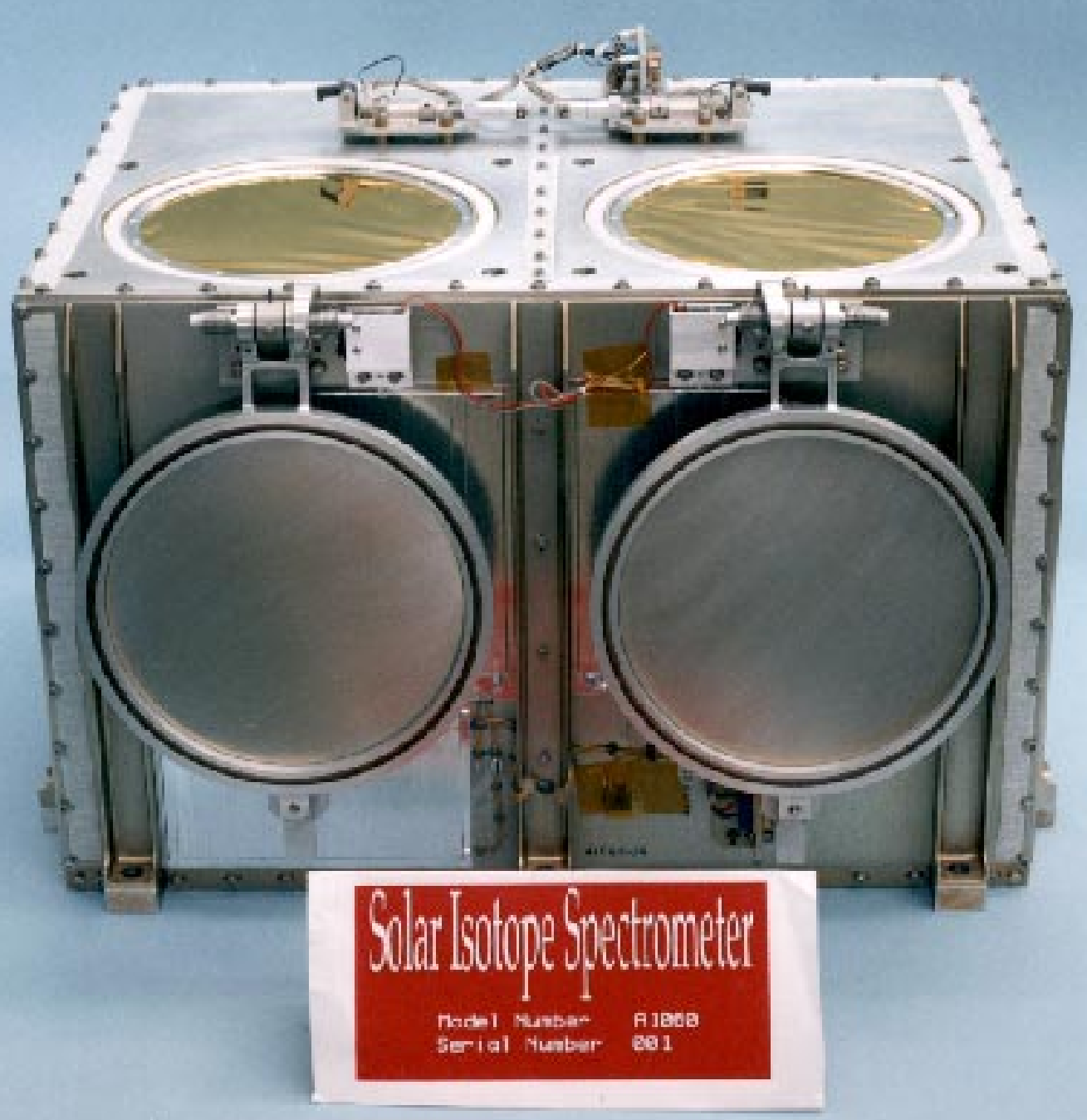

Figure 1. 


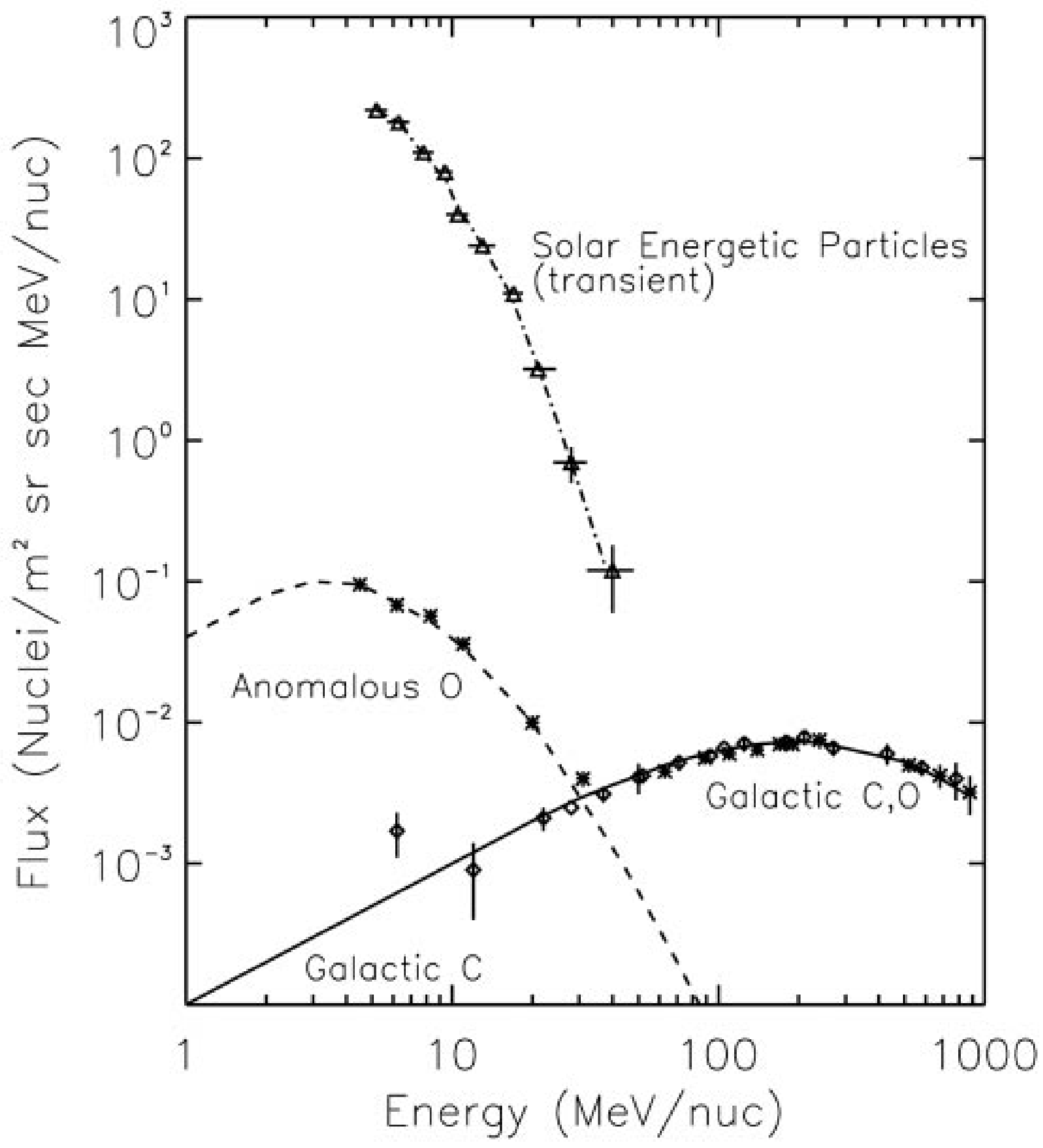




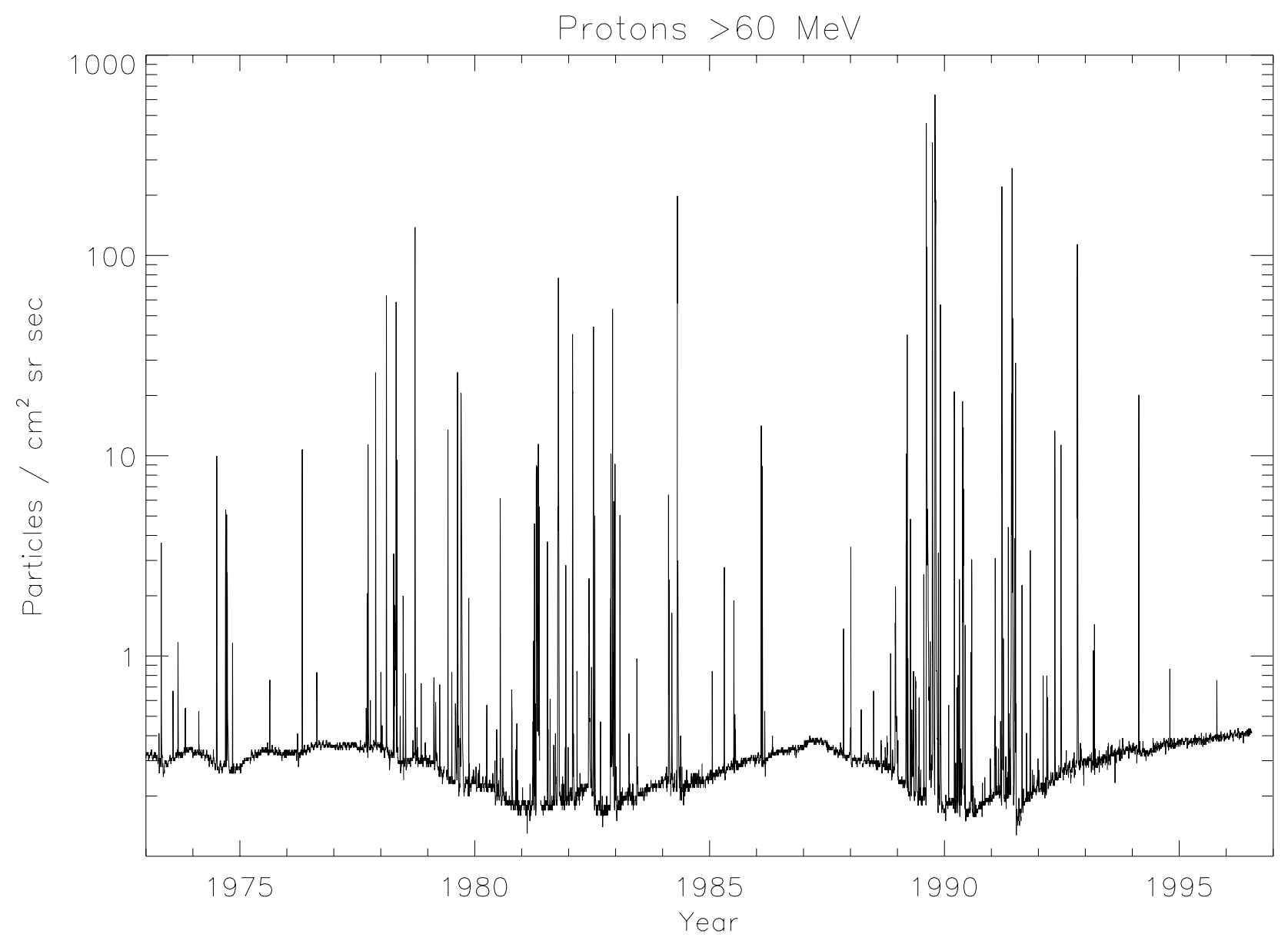



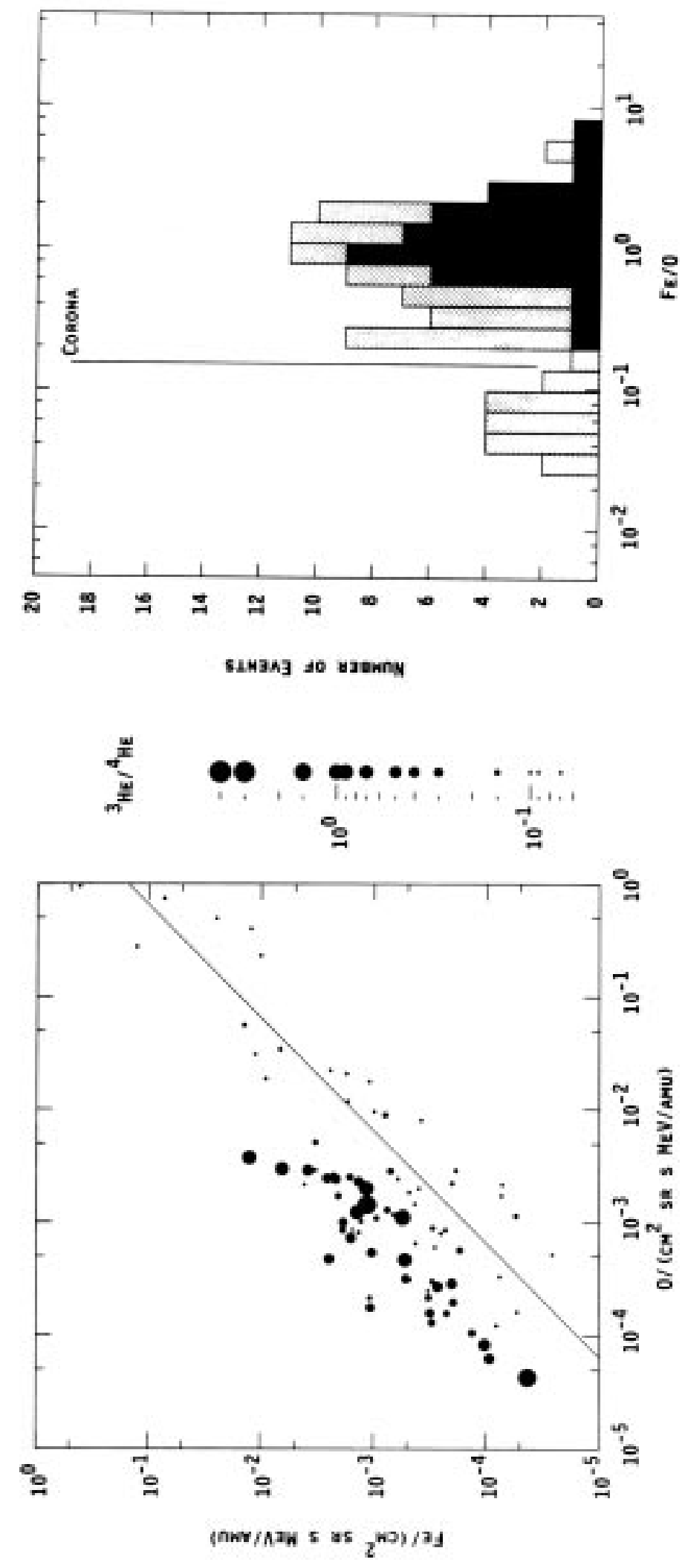

Figure 4. 


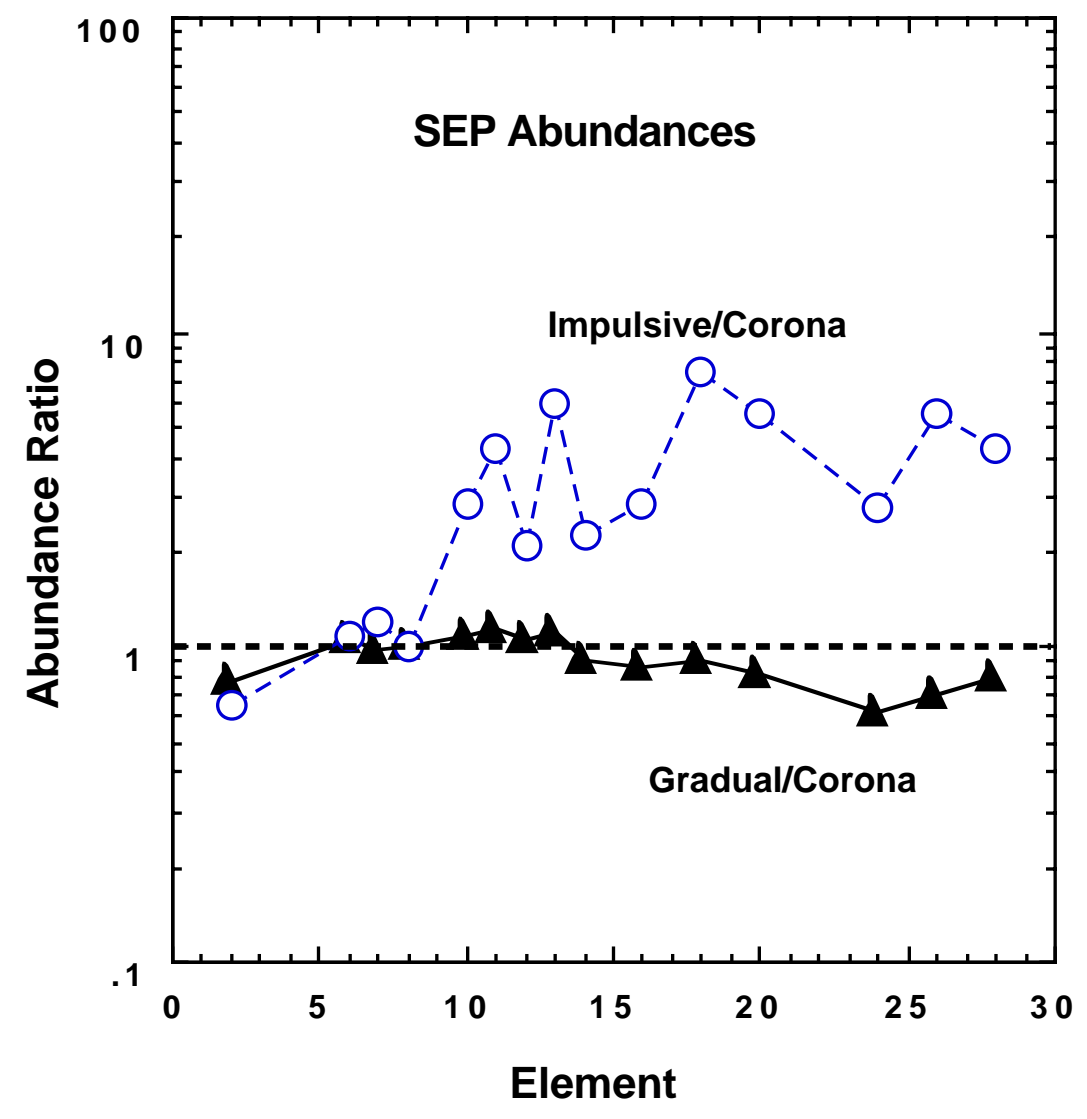




\section{SOLAR CORONAL ISOTOPE ABUNDANCES}

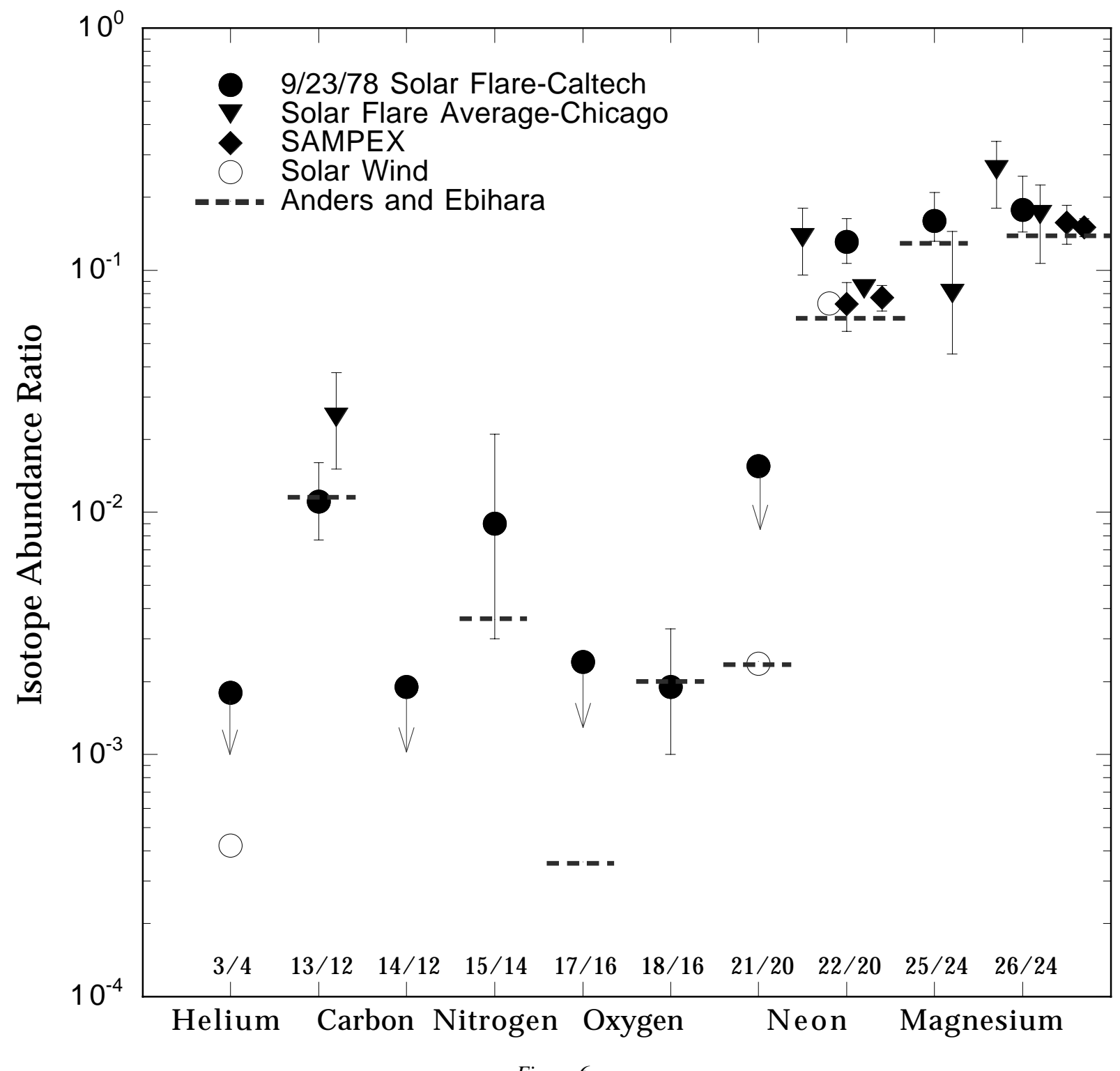

Figure 6. 
Estimated Number of Solar Energetic Particles Collected

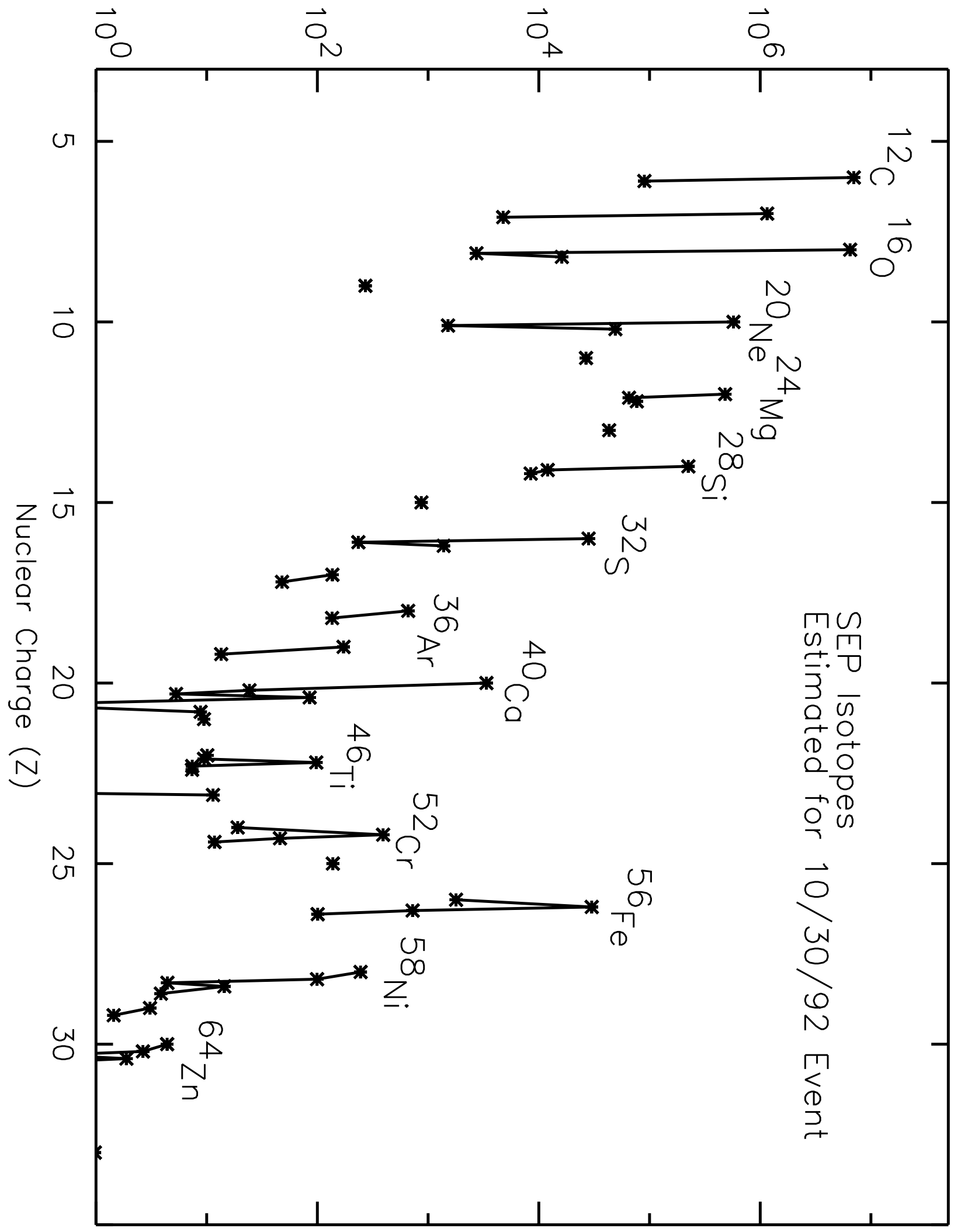

Figure 7. 


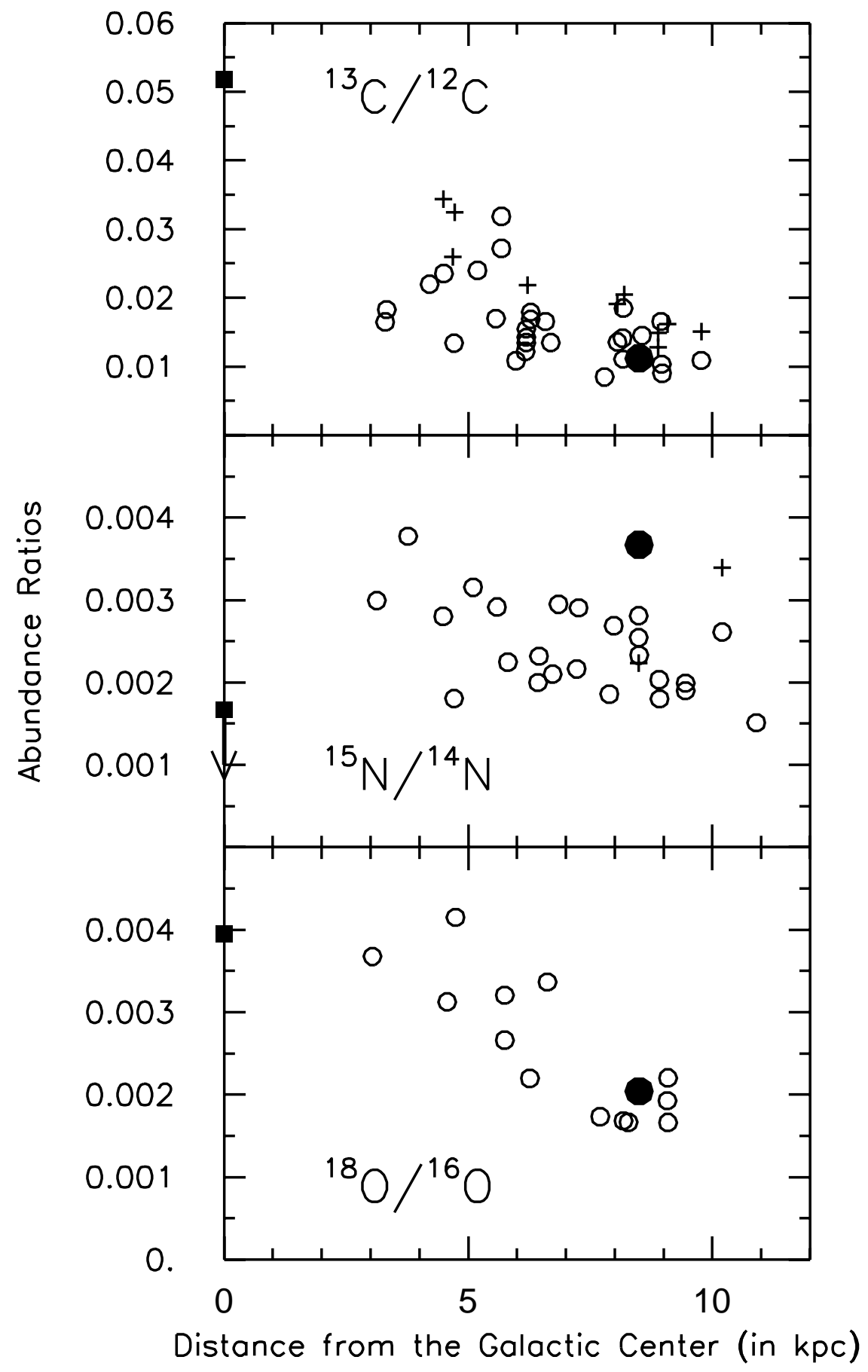

Figure 8. 


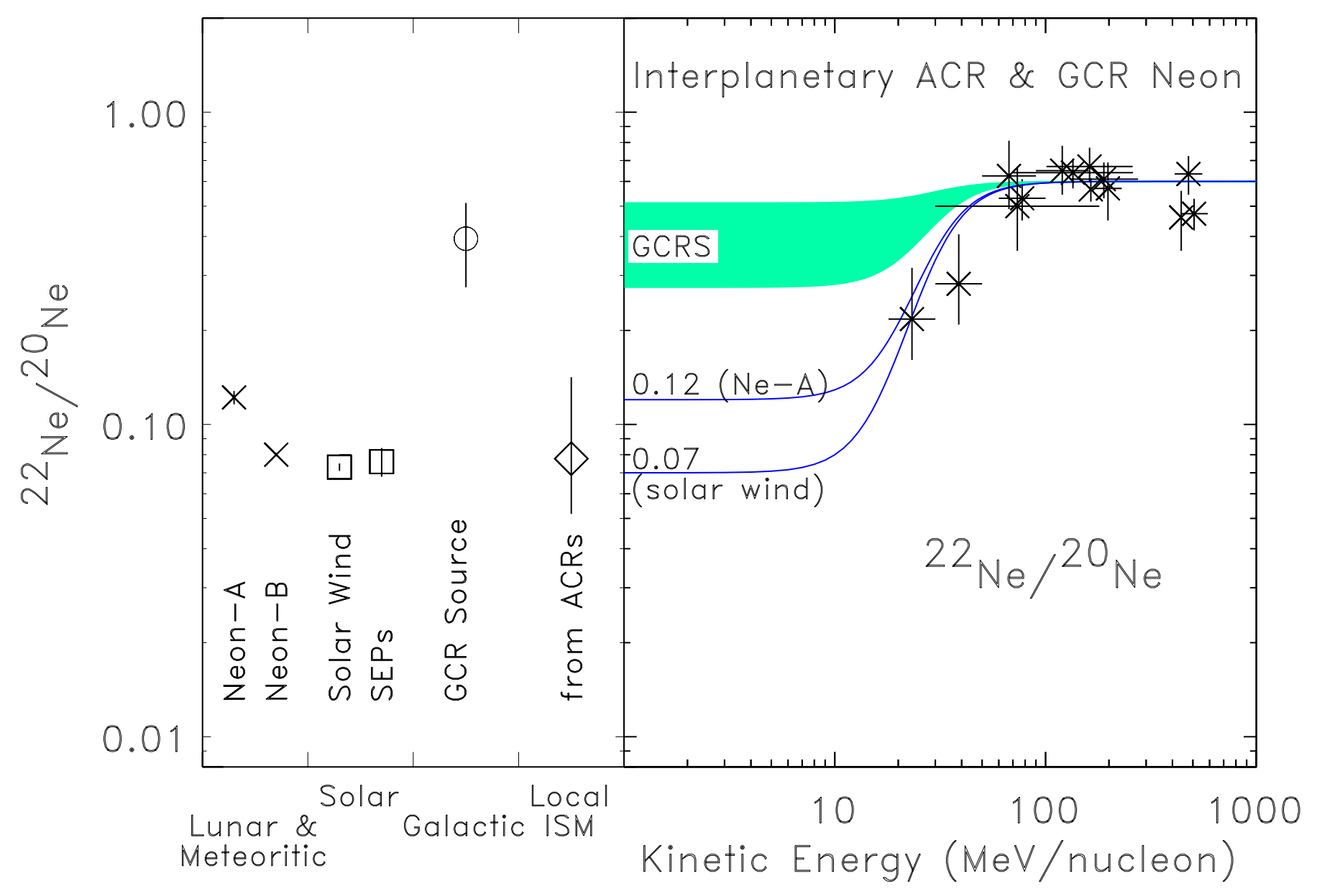

Figure 9. 


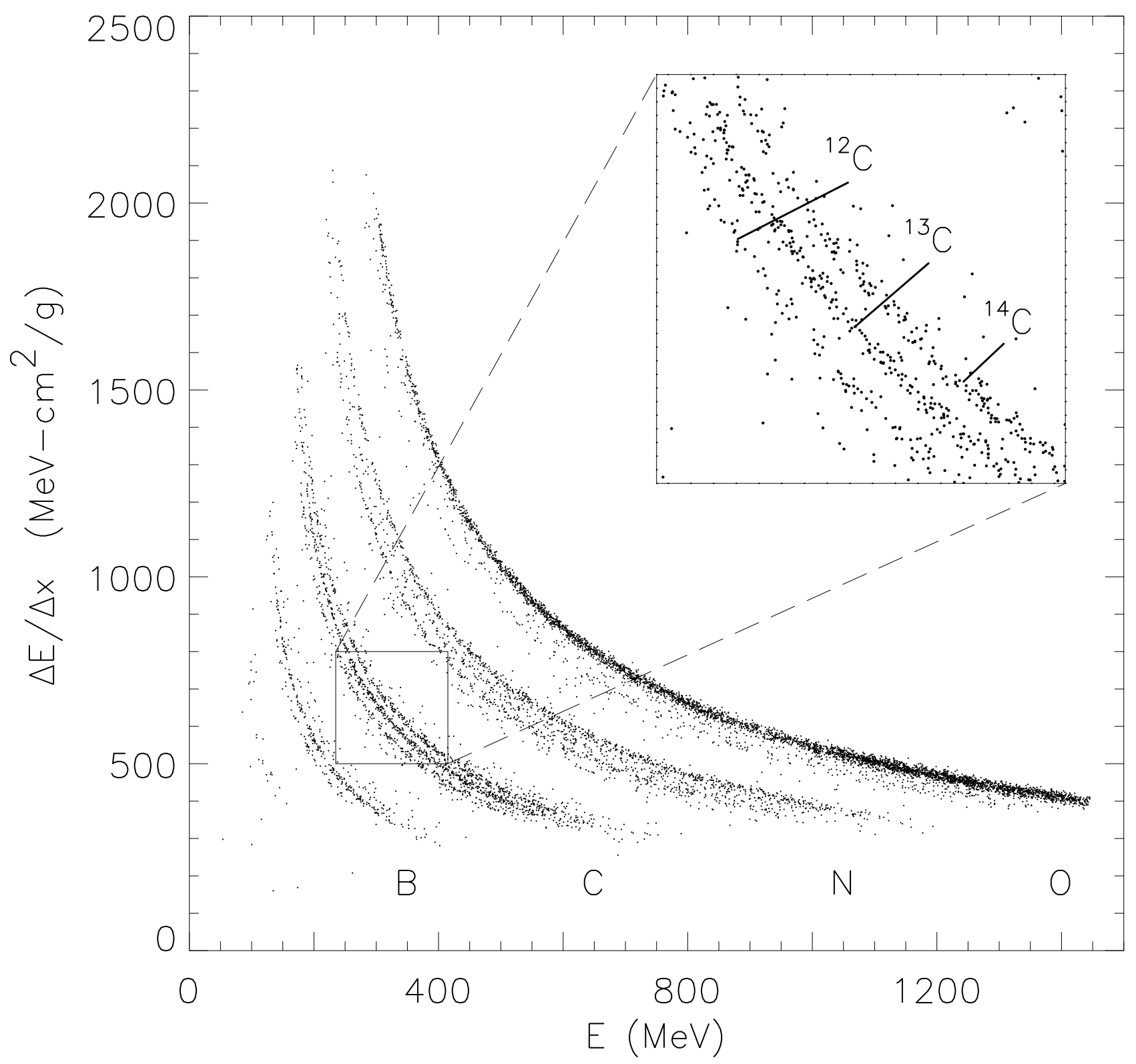

Figure 10. 


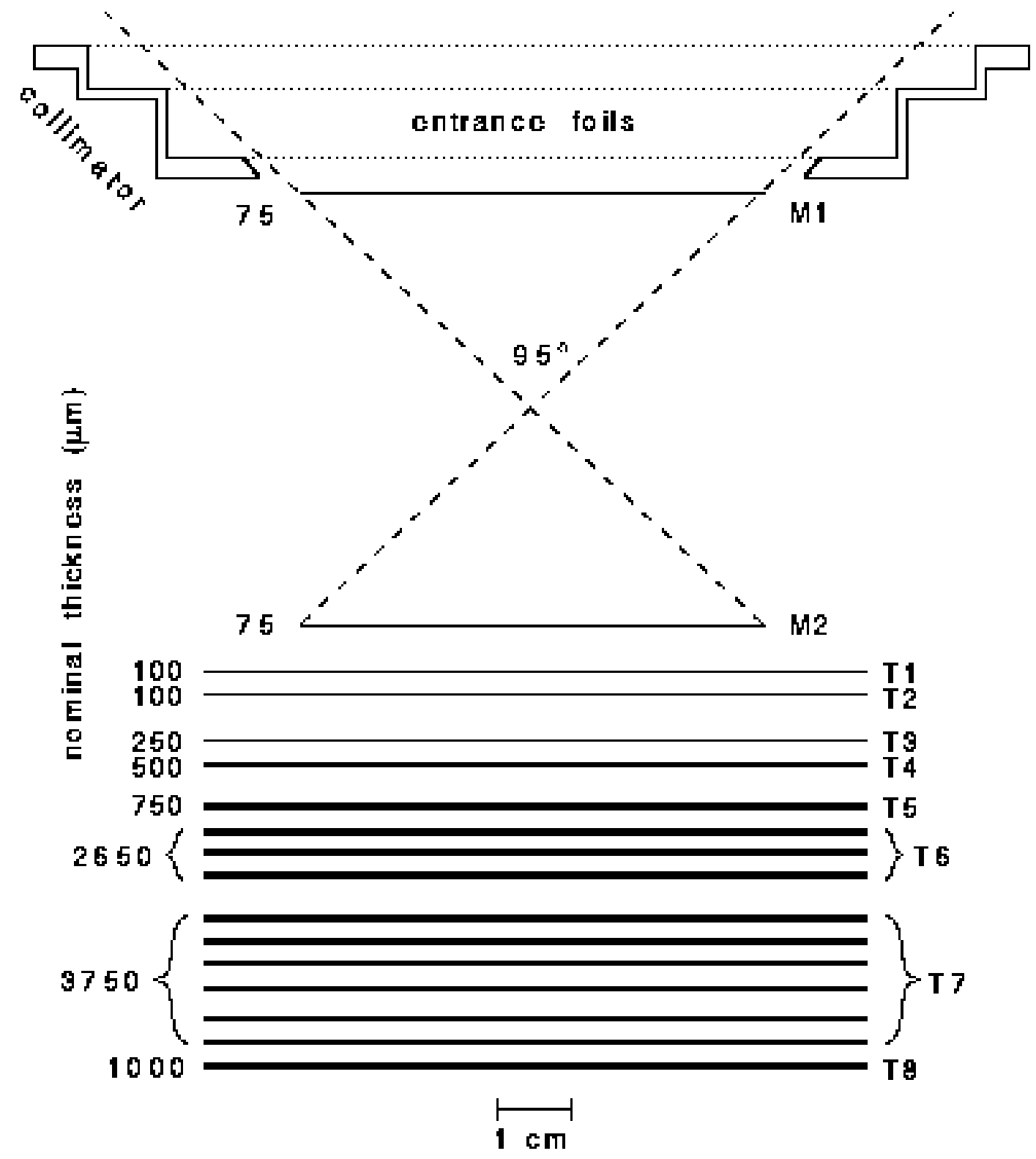

Figure 11. 


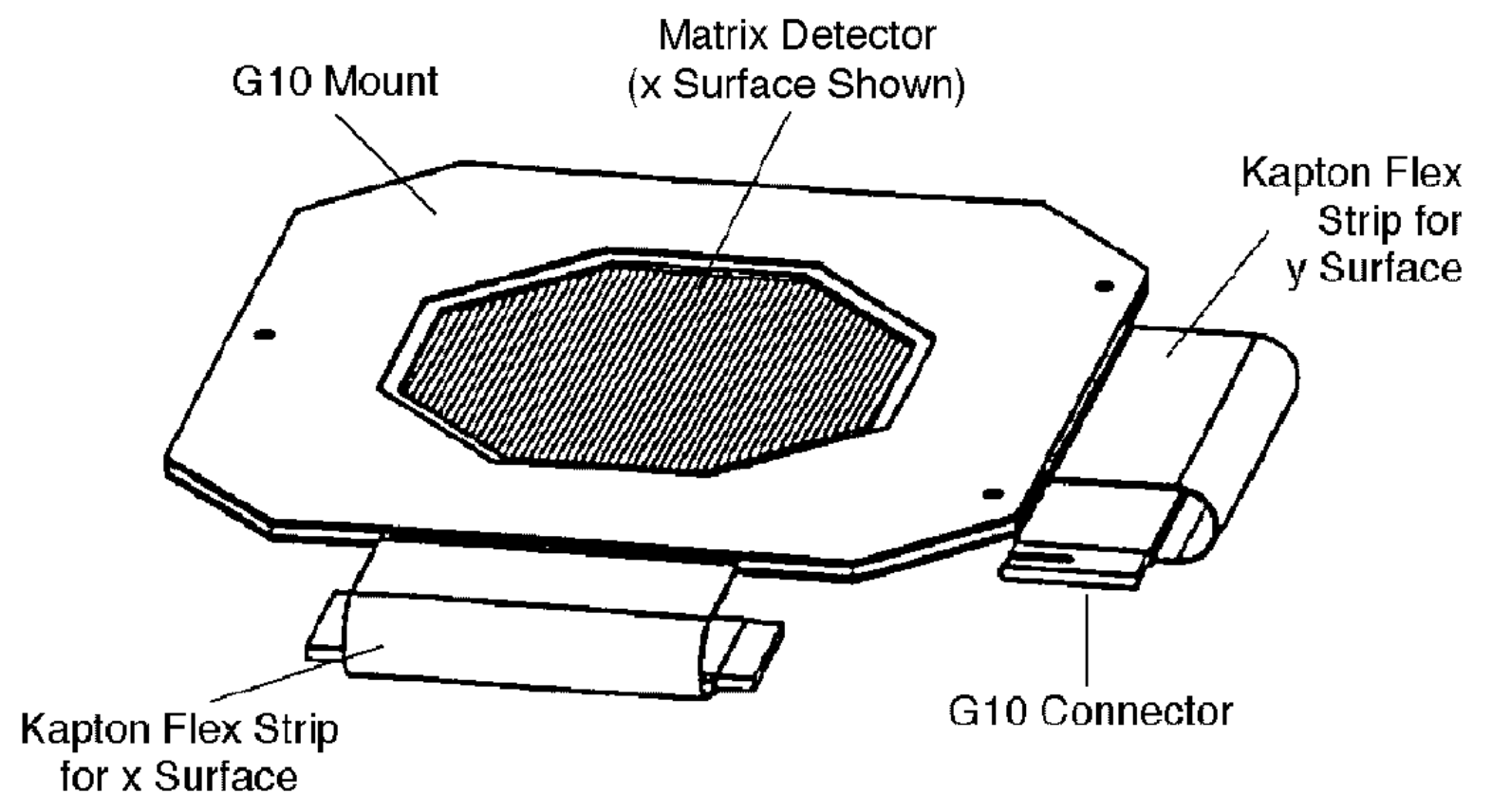

Figure 12. 
SOLAR ISOTOPE SPECTROMETER (SIS) Block Diagram

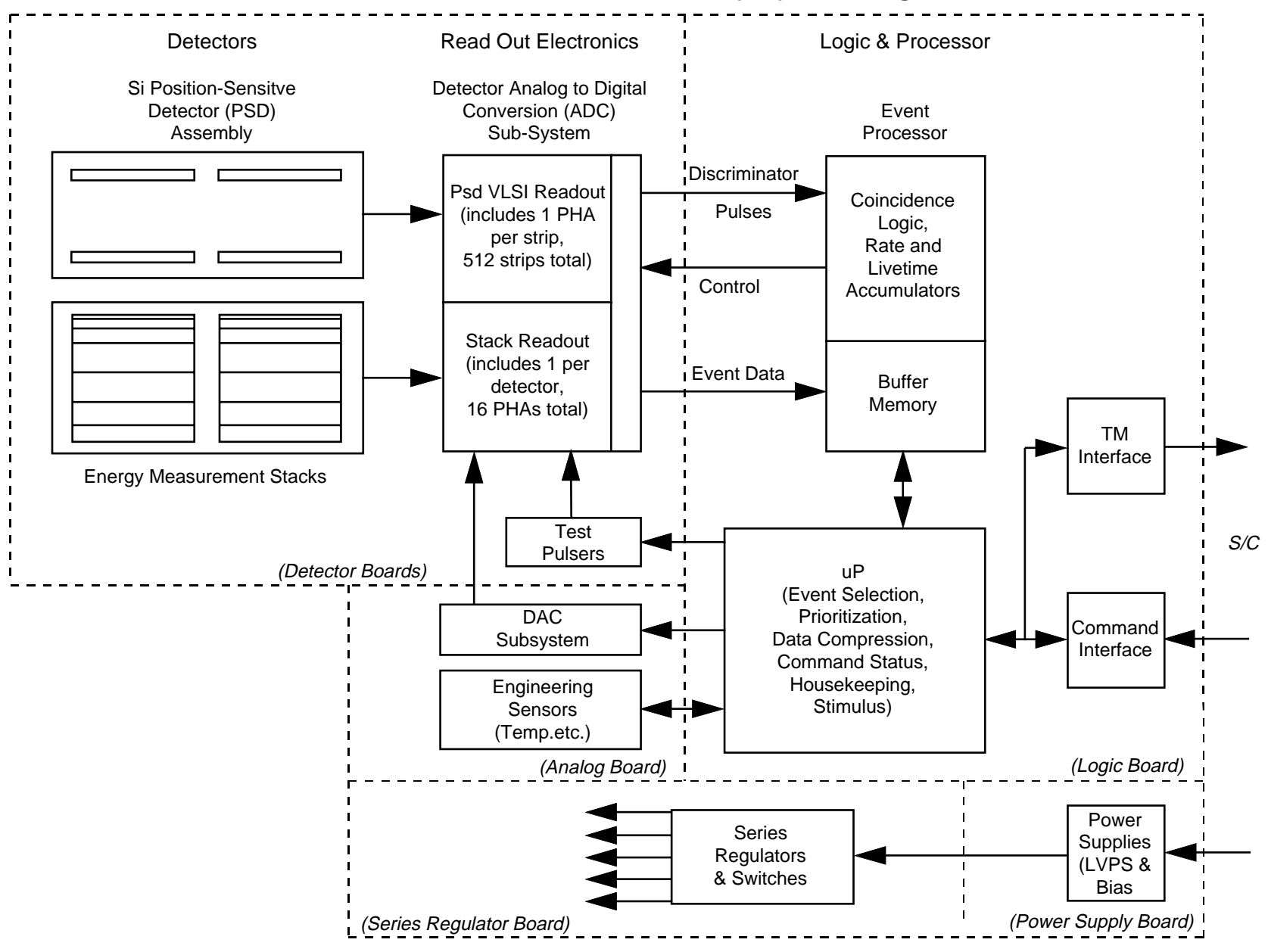

Figure 13. 


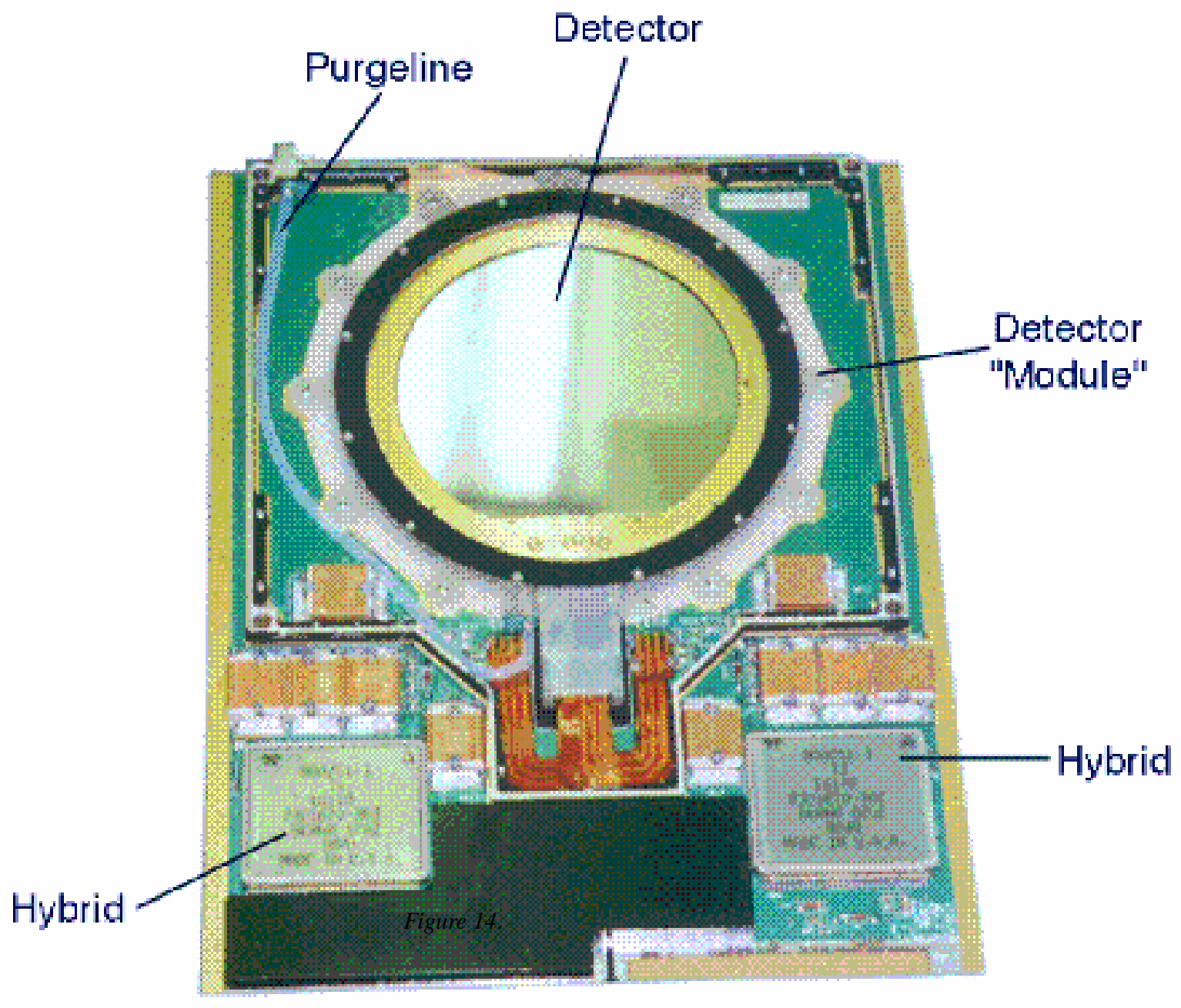




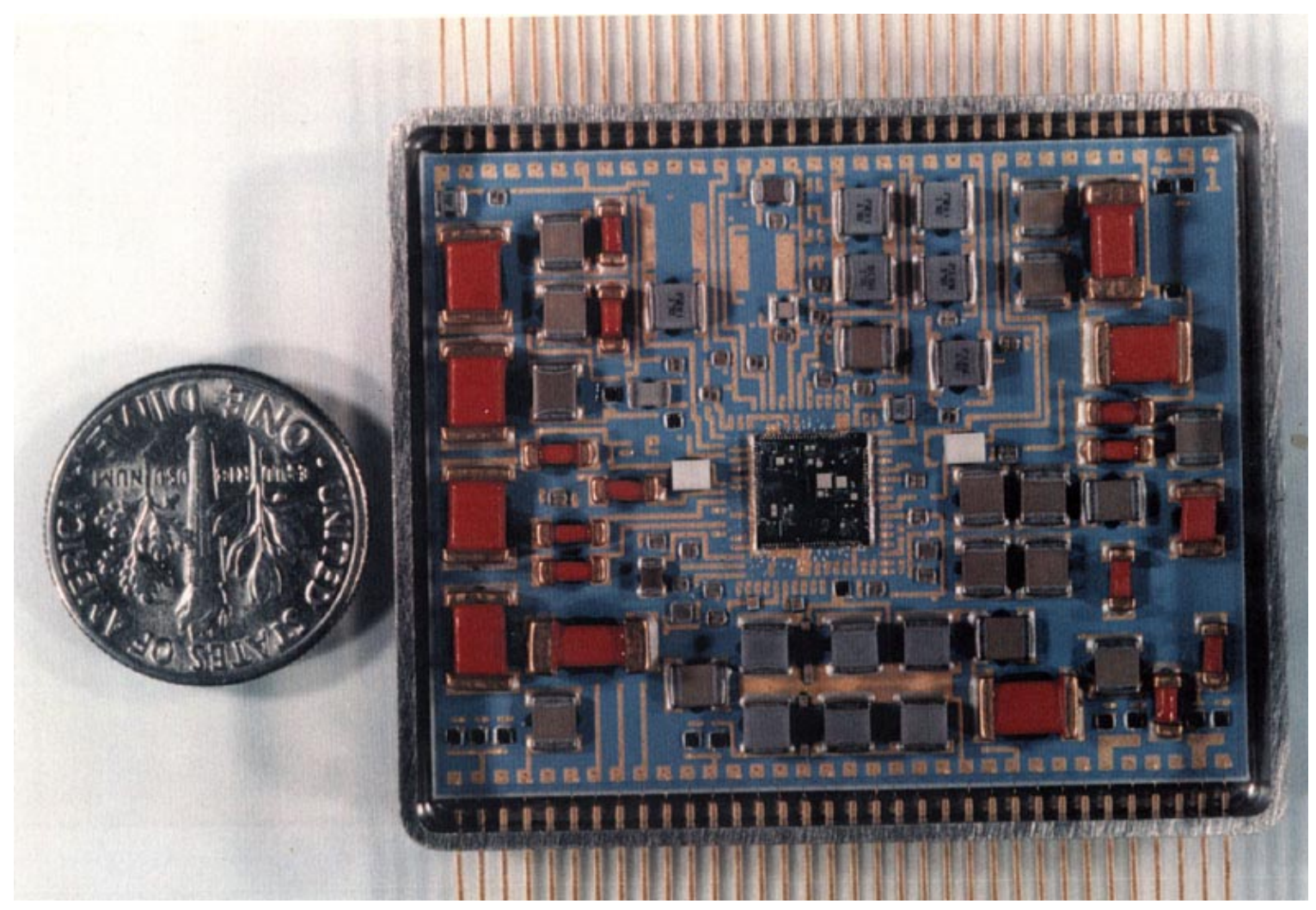

Figure 15. 


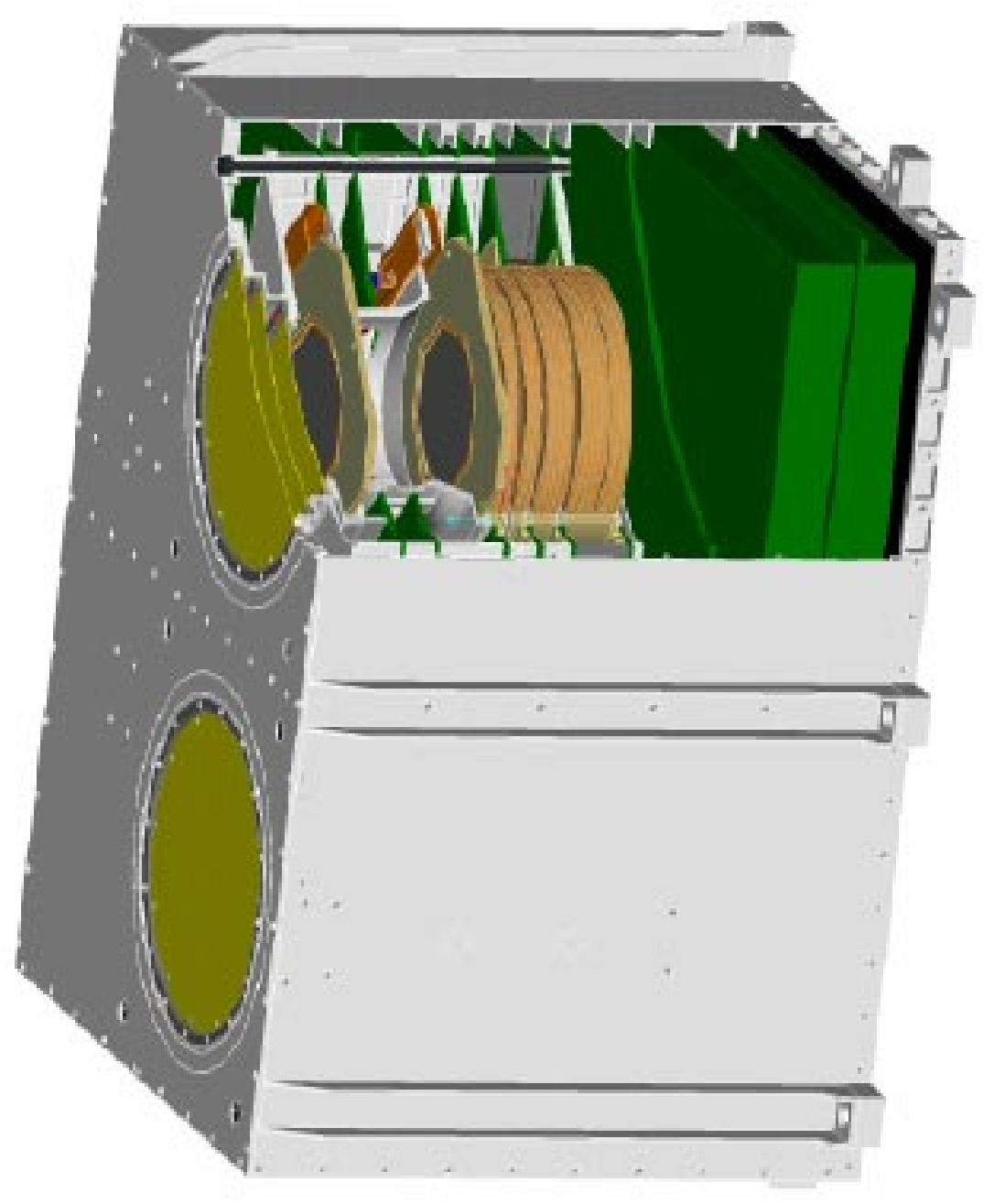

Figure 16. 


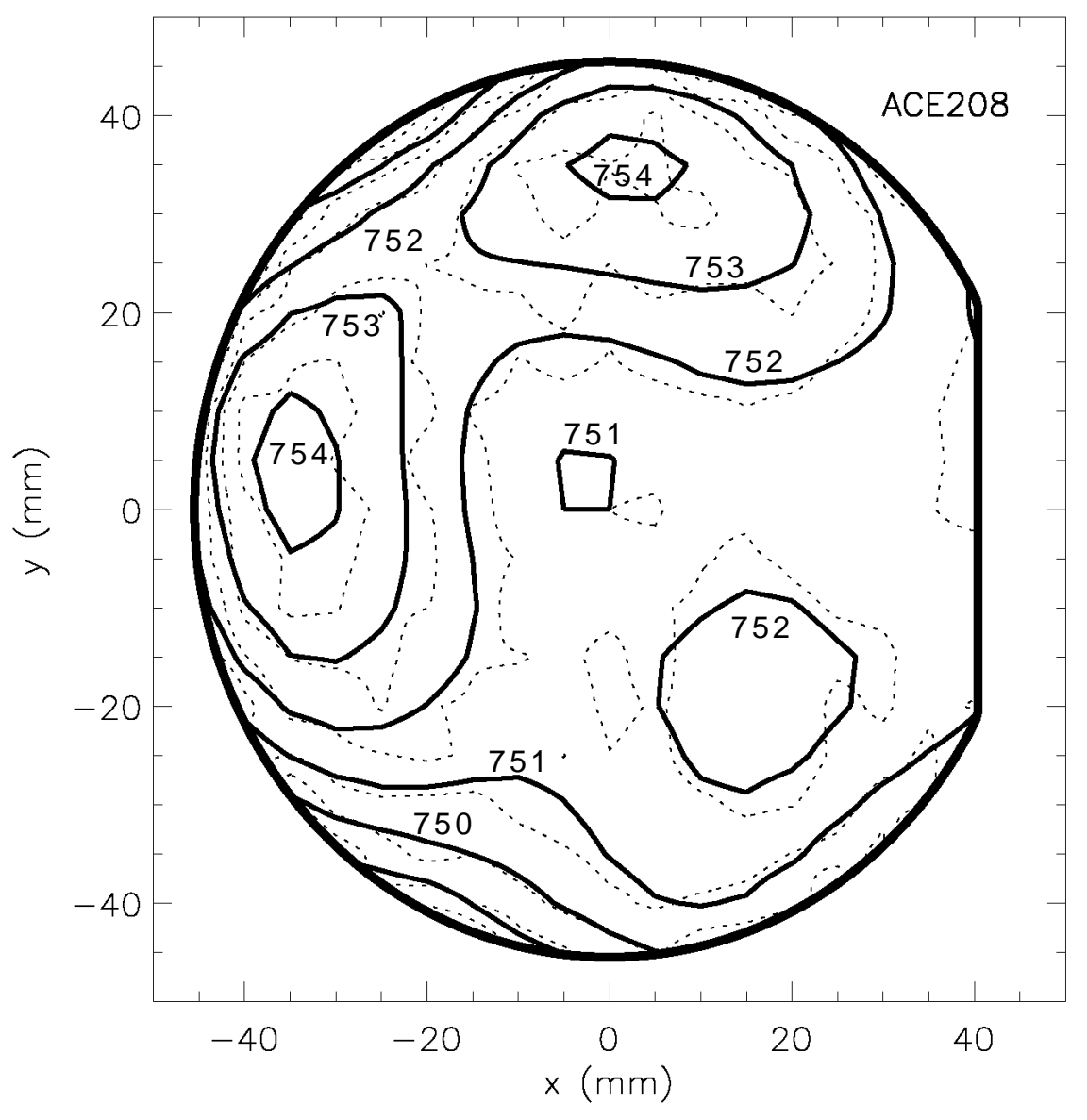

Figure 17. 


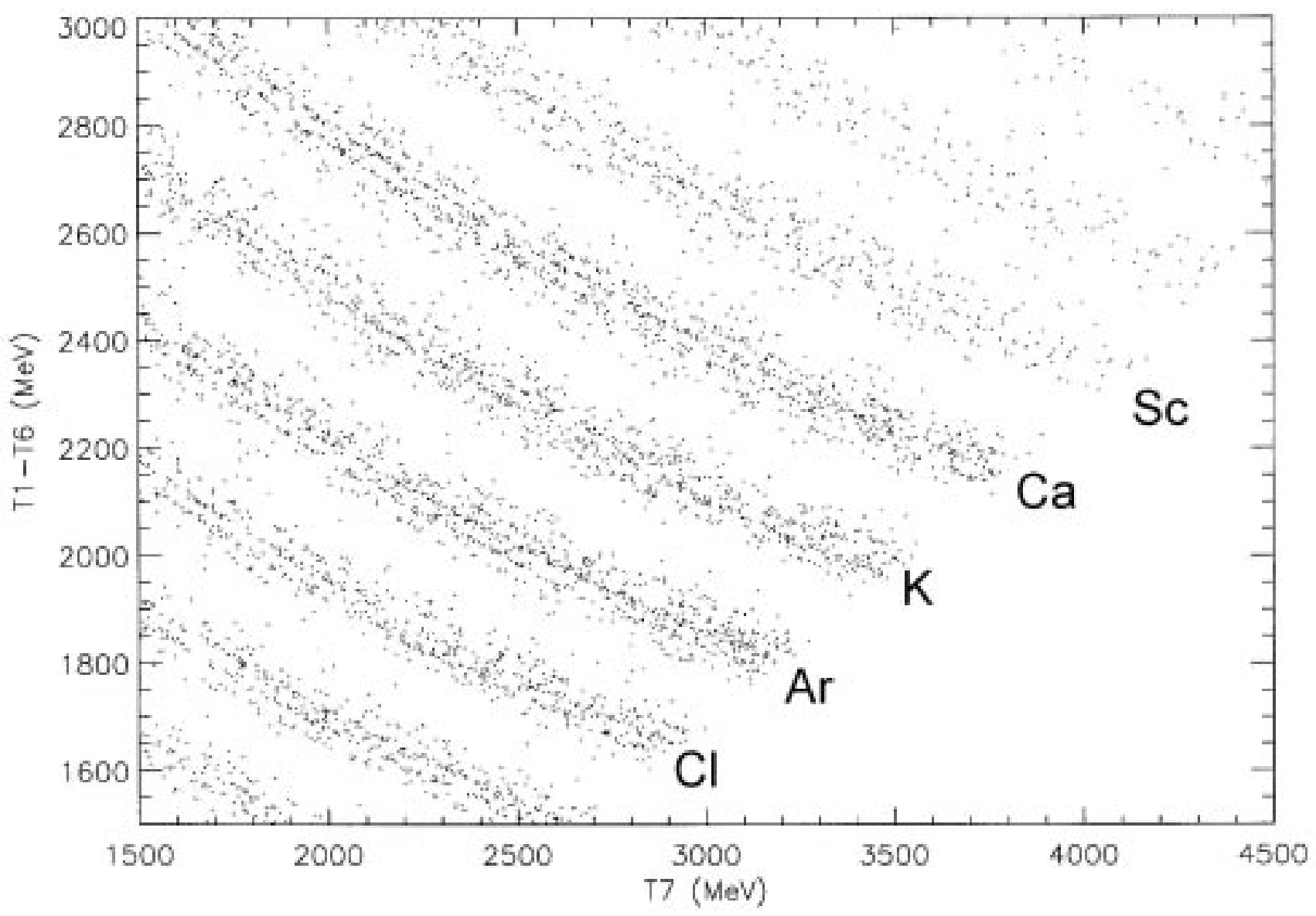

Figure 18. 


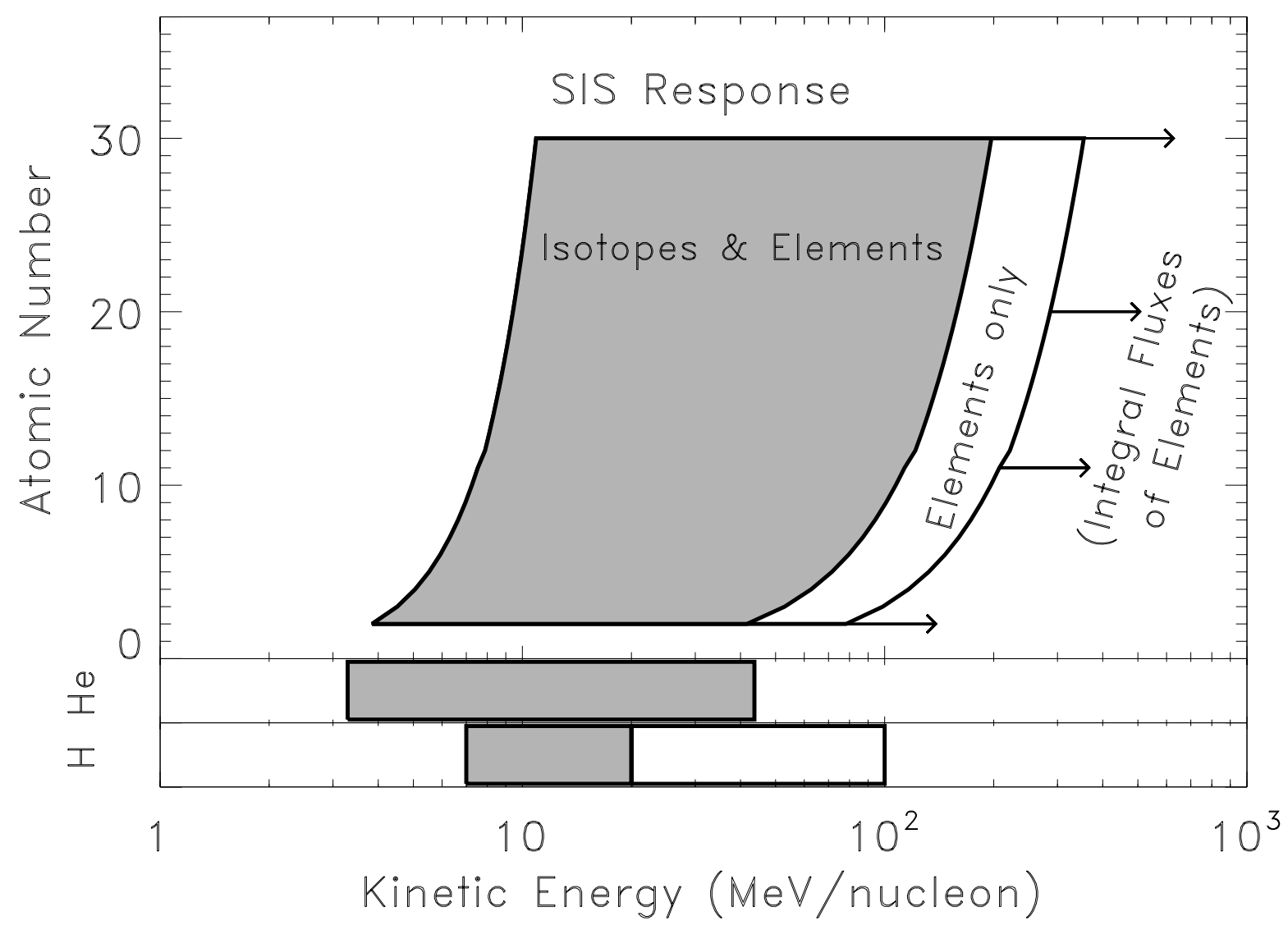

Figure 19. 
Address for correspondence: R. A. Mewaldt, Mail Code 220-47, Caltech, Pasadena CA 91125. 